\title{
The Feynman path integral approach to atomic interferometry. A tutorial
}

\author{
Pippa Storey and Claude Cohen-Tannoudji \\ Collège de France and Laboratoire Kastler Brossel de l'Ecole Normale Supérieure (*) \\ 24 rue Lhomond, 75231 Paris Cedex 05, France
}

(Received 22 September 1994, accepted 26 September 1994)

\begin{abstract}
Many problems of current interest in atomic interferometry lend themselves to a path integral treatment. We present a practical guide to solving such problems, taking as examples the gravitational experiments of Kasevich and $\mathrm{Chu}$, and the atomic equivalents of the Sagnac and Aharonov-Bohm effects.
\end{abstract}

\section{Introduction.}

Atomic interferometry is a new and rapidly-developing field of research, concerned with physical phenomena in which the wave-nature of neutral atoms plays an important role [1]. The wide variety of internal degrees of freedom of an atom opens up new possibilities for investigation which do not exist in the more traditional types of interferometry using photons, electrons and neutrons.

The development of atomic interferometry has been aided by recent technical advances, particularly in the manipulation of atoms. New mechanisms for slowing, deflecting, cooling and trapping atoms allow control of both their position and momentum. Also important has been the birth of "atomic optics", a range of mechanisms providing the equivalent of mirrors, beamsplitters and lenses for atoms. Recently it has been pointed out that certain high-resolution spectroscopy techniques which avoid the Doppler effect amount to realizing an atomic interferometer [2]. These methods have since been adapted to measure inertial fields (due to rotation and gravitation) by atomic interferometry.

The situation encountered in atomic interferometry experiments is often close to the classical limit. When this is the case a path integral approach to the analysis is very appropriate since it reduces to a calculation of integrals along classical paths. Further simplifications can be made if the Lagrangian is quadratic, as is true for a particle in a gravitational field or a rotating

(*) The Laboratoire Kastler Brossel is associated with the CNRS and the Universite Pierre et Marie Curie. 
reference frame. A simple perturbative treatment is also possible. The path integral method allows new physical insight, and links the traditional formulation of quantum mechanics with the Huygens-Fresnel principle of wave mechanics.

The main body of the article consists of two parts, the first being a presentation of the path integral method, and the second consisting of applications to various examples in atomic interferometry. The first part begins with a brief review of Lagrangian dynamics. The quantum propagator is introduced, and a simple expression for it derived for the case of quadratic Lagrangians. The quantum propagator is then applied to the propagation of wavefunctions, and a practical perturbative approach is obtained. This is used to calculate the phase difference between the two arms of an interferometer. In the second part the theory is applied to the following systems: a free particle, a two-level atom crossing a laser wave, a particle in a gravitational field (including an analysis of the experiment by Kasevich and Chu), a particle in a rotating frame, and the atomic equivalents of the Aharonov-Bohm effects.

\section{Path integral method.}

1.1 Review of Classical Lagrangian dynamics. - In this section we review some elements of classical Lagrangian dynamics [3], beginning with the principle of least action and the Lagrange equations. We define the momentum and the Hamiltonian, and state their relationship to the partial derivatives of the classical action. Using this formalism we obtain an expansion of the classical action about an initial position, which will be used in the following sections.

1.1.1 Paths in space-time. - We consider a classical particle travelling from the point $z_{\mathrm{a}} t_{\mathrm{a}}$ to the point $z_{\mathrm{b}} t_{\mathrm{b}}$ as shown in figure 1 . There exists an infinity of possible spacetime paths $\left(\Gamma, \Gamma^{\prime} ..\right)$ linking these two points, each path being described by a function $z(t)$ such that $z\left(t_{\mathrm{a}}\right)=z_{\mathrm{a}}$ and $z\left(t_{\mathrm{b}}\right)=z_{\mathrm{b}}$. Of all the possible paths, there is one which is actually taken by the particle and which can be determined from the Lagrangian of the system. The Lagrangian $L$ is a certain function of position $z$ and velocity $\dot{z}$, which for a particle of mass $M$ in a potential $V(z)$ is

$$
L(z, \dot{z})=\frac{1}{2} M \dot{z}^{2}-V(z)
$$

1.1.2 Principle of least action. - The actual path taken by a classical particle is the one for which the action is extremal. The action is defined as the integral of the Lagrangian $L(z, \dot{z})$ over the given path $z(t)$

$$
S_{\Gamma}=\int_{t_{\mathrm{a}}}^{t_{\mathrm{b}}} \mathrm{d} t L[z(t), \dot{z}(t)] .
$$

We will denote by $\Gamma_{\mathrm{cl}}$ the actual classical path, for which the action is extremal, and by $S_{\mathrm{cl}}$ the corresponding value of the action. This "classical" action is a function only of the endpoints of the path, $S_{\mathrm{cl}} \equiv S_{\mathrm{cl}}\left(z_{\mathrm{b}} t_{\mathrm{b}}, z_{\mathrm{a}} t_{\mathrm{a}}\right)$.

1.1.3 Lagrange equations. - The Lagrange equations are a differential form of the principle of least action (see Appendix A.1), and describe the dynamics of the system. They can be written as

$$
\frac{\partial L}{\partial z}-\frac{\mathrm{d}}{\mathrm{d} t} \frac{\partial L}{\partial \dot{z}}=0
$$

and are equivalent to the well-known Newton equation

$$
\mathbf{F}=M \mathbf{a}
$$

relating the force $\mathbf{F}$ to the acceleration a. 


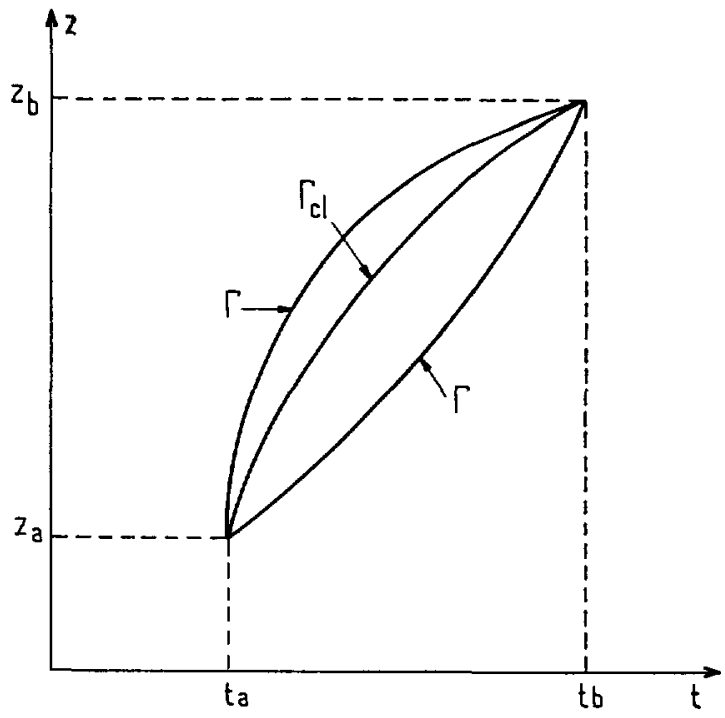

Fig. 1. - Possible paths in spacetime connecting the initial point $z_{\mathrm{a}} t_{\mathrm{a}}$ to the final point $z_{\mathrm{b}} t_{\mathrm{b}} . \Gamma_{\mathrm{cl}}$ is the actual classical path, solution of the equations of motion.

1.1.4 Partial derivatives of the classical action. - The partial derivatives of the classical action are calculated in Appendix A.2. Defining momentum as

$$
p \equiv \frac{\partial L}{\partial \dot{z}},
$$

the partial derivatives with respect to the initial and final positions can be written as

$$
\begin{aligned}
& \frac{\partial}{\partial z_{\mathrm{a}}} S_{\mathrm{cl}}\left(z_{\mathrm{b}} t_{\mathrm{b}}, z_{\mathrm{a}} t_{\mathrm{a}}\right)=-p_{\mathrm{a}} \\
& \frac{\partial}{\partial z_{\mathrm{b}}} S_{\mathrm{cl}}\left(z_{\mathrm{b}} t_{\mathrm{b}}, z_{\mathrm{a}} t_{\mathrm{a}}\right)=p_{\mathrm{b}},
\end{aligned}
$$

where $p_{\mathrm{a}}\left(p_{\mathrm{b}}\right)$ is the momentum of the particle along the classical path $\Gamma_{\mathrm{cl}}$ at the point $z_{\mathrm{a}} t_{\mathrm{a}}$ $\left(z_{\mathrm{b}} t_{\mathrm{b}}\right)$.

Similarly, the Hamiltonian is defined by

$$
H \equiv p \dot{z}-L,
$$

and the partial derivatives of classical action with respect to the initial and final times are given by

$$
\begin{aligned}
& \frac{\partial}{\partial t_{\mathrm{a}}} S_{\mathrm{cl}}\left(z_{\mathrm{b}} t_{\mathrm{b}}, z_{\mathrm{a}} t_{\mathrm{a}}\right)=H_{\mathrm{a}} \\
& \frac{\partial}{\partial t_{\mathrm{b}}} S_{\mathrm{cl}}\left(z_{\mathrm{b}} t_{\mathrm{b}}, z_{\mathrm{a}} t_{\mathrm{a}}\right)=-H_{\mathrm{b}},
\end{aligned}
$$

where $H_{\mathrm{a}}\left(H_{\mathrm{b}}\right)$ is the Hamiltonian of the particle along the classical path $\Gamma_{\mathrm{cl}}$ at the point $z_{\mathrm{a}} t_{\mathrm{a}}$ $\left(z_{\mathrm{b}} t_{\mathrm{b}}\right)$. 
Using the values of the partial derivatives, we can obtain the differential of the classical action. Keeping the initial spacetime point fixed, the variation in the classical action due to a change in the final spacetime point $(z, t)$ is

$$
\mathrm{d} S_{\mathrm{cl}}=\frac{\partial S_{\mathrm{cl}}}{\partial z} \mathrm{~d} z+\frac{\partial S_{\mathrm{cl}}}{\partial t} \mathrm{~d} t=p \mathrm{~d} z-H \mathrm{~d} t
$$

This gives an alternative expression for the classical action

$$
S_{\mathrm{cl}}=\int_{\Gamma_{\mathrm{cl}}}(p \mathrm{~d} z-H \mathrm{~d} t)
$$

We also obtain an expansion for the classical action about the initial position $z_{\mathrm{a}}$ (holding the initial time $t_{\mathrm{a}}$ and the final coordinates $z_{\mathrm{b}} t_{\mathrm{b}}$ constant).

$$
\begin{aligned}
S_{\mathrm{cl}}\left(z_{\mathrm{b}}, t_{\mathrm{b}} ; z_{\mathrm{a}}+\zeta, t_{\mathrm{a}}\right) & =S_{\mathrm{cl}}\left(z_{\mathrm{b}} t_{\mathrm{b}}, z_{\mathrm{a}} t_{\mathrm{a}}\right)+\zeta \frac{\partial}{\partial z_{\mathrm{a}}} S_{\mathrm{cl}}\left(z_{\mathrm{b}} t_{\mathrm{b}}, z_{\mathrm{a}} t_{\mathrm{a}}\right)+\frac{1}{2} \zeta^{2} \frac{\partial^{2}}{\partial z_{\mathrm{a}}^{2}} S_{\mathrm{cl}}\left(z_{\mathrm{b}} t_{\mathrm{b}}, z_{\mathrm{a}} t_{\mathrm{a}}\right)+ \\
& =S_{\mathrm{cl}}\left(z_{\mathrm{b}} t_{\mathrm{b}}, z_{\mathrm{a}} t_{\mathrm{a}}\right)-\zeta p_{\mathrm{a}}-\frac{1}{2} \zeta^{2} \frac{\partial p_{\mathrm{a}}}{\partial z_{\mathrm{a}}}+
\end{aligned}
$$

1.2 The quantum Propagator. - In this section we define the quantum propagator. The composition property which it obeys is used to explain intuitively the equivalence between this usual definition and Feynman's formulation. A simple expression for the propagator is then obtained for the case of quadratic Lagrangians.

1.2.1 Definition of the quantum propagator. - The state of a quantum system at a final time $t_{\mathrm{b}}$ is determined by its state at an earlier time $t_{\mathrm{a}}$ through the evolution operator $U$

$$
\left|\psi\left(t_{\mathrm{b}}\right)\right\rangle=U\left(t_{\mathrm{b}}, t_{\mathrm{a}}\right)\left|\psi\left(t_{\mathrm{a}}\right)\right\rangle
$$

The final wavefunction, given by the projection of the final state onto the position basis, is therefore

$$
\begin{aligned}
\psi\left(z_{\mathrm{b}}, t_{\mathrm{b}}\right) & =\left\langle z_{\mathrm{b}} \mid \psi\left(t_{\mathrm{b}}\right)\right\rangle=\left\langle z_{\mathrm{b}}\left|U\left(t_{\mathrm{b}}, t_{\mathrm{a}}\right)\right| \psi\left(t_{\mathrm{a}}\right)\right\rangle \\
& =\int \mathrm{d} z_{\mathrm{a}}\left\langle z_{\mathrm{b}}\left|U\left(t_{\mathrm{b}}, t_{\mathrm{a}}\right)\right| z_{\mathrm{a}}\right\rangle\left\langle z_{\mathrm{a}} \mid \psi\left(t_{\mathrm{a}}\right)\right\rangle \\
& =\int \mathrm{d} z_{\mathrm{a}} K\left(z_{\mathrm{b}} t_{\mathrm{b}}, z_{\mathrm{a}} t_{\mathrm{a}}\right) \psi\left(z_{\mathrm{a}}, t_{\mathrm{a}}\right)
\end{aligned}
$$

where the function $K$ has been defined as

$$
K\left(z_{\mathrm{b}} t_{\mathrm{b}}, z_{\mathrm{a}} t_{\mathrm{a}}\right) \equiv\left\langle z_{\mathrm{b}}\left|U\left(t_{\mathrm{b}}, t_{\mathrm{a}}\right)\right| z_{\mathrm{a}}\right\rangle
$$

$K$ is known as the quantum propagator, and denotes the amplitude for the particle to arrive at point $z_{\mathrm{b}} t_{\mathrm{b}}$ given that it starts from point $z_{\mathrm{a}} t_{\mathrm{a}}$.

Equation (15) reveals an analogy between quantum wavefunction propagation and the FresnelHuygens principle: the value of the wavefunction at the point $z_{\mathrm{b}} t_{\mathrm{b}}$ is the superposition of all wavelets radiated by the "point sources" $z_{\mathrm{a}} t_{\mathrm{a}}, z_{\mathrm{a}}^{\prime} t_{\mathrm{a}}, z_{a}^{\prime \prime} t_{\mathrm{a}} \ldots$ (see Fig. 2). 


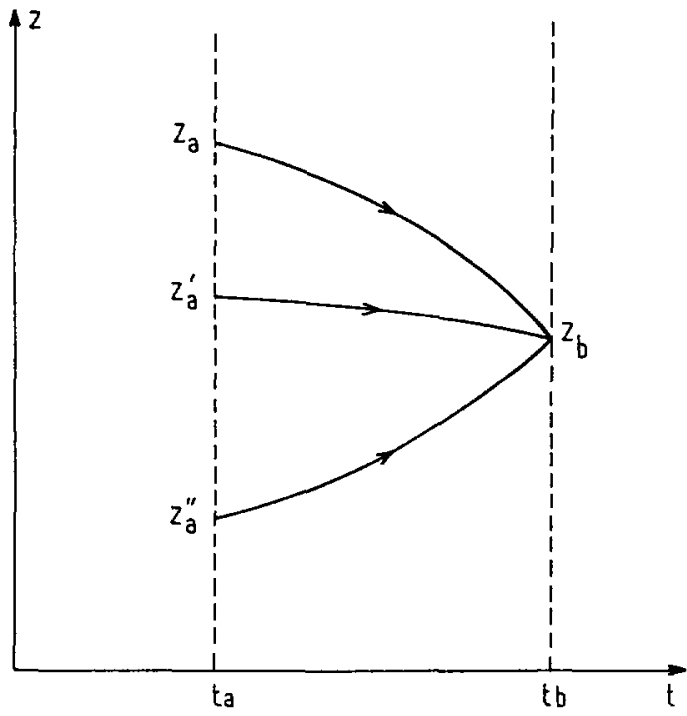

Fig. 2. - The wavefunction at $z_{\mathrm{b}} t_{\mathrm{b}}$ is a superposition of contributions from all the point sources $z_{\mathrm{a}} t_{\mathrm{a}}$, $z_{\mathrm{a}}^{\prime} t_{\mathrm{a}}, z_{\mathrm{a}}^{\prime \prime} t_{\mathrm{a}} \ldots$

1.2.2 The composition property of the quantum propagator. - The composition property of the quantum propagator can be derived using the following relation for the evolution operator

$$
U\left(t_{\mathrm{b}}, t_{\mathrm{a}}\right)=U\left(t_{\mathrm{b}}, t_{\mathrm{c}}\right) U\left(t_{\mathrm{c}}, t_{\mathrm{a}}\right),
$$

where $t_{\mathrm{c}}$ is any intermediate time between $t_{\mathrm{a}}$ and $t_{\mathrm{b}}$. This equation simply states that the evolution of an arbitrary state from an initial time to a final time may be calculated in two stages: the evolution of that state from the initial time to any intermediate time, followed by the evolution of the resulting intermediate state from the intermediate to the final time.

Substituting the above equation into the definition (16) of the quantum propagator gives

$$
\begin{aligned}
K\left(z_{\mathrm{b}} t_{\mathrm{b}}, z_{\mathrm{a}} t_{\mathrm{a}}\right) & =\left\langle z_{\mathrm{b}}\left|U\left(t_{\mathrm{b}}, t_{\mathrm{c}}\right) U\left(t_{\mathrm{c}}, t_{\mathrm{a}}\right)\right| z_{\mathrm{a}}\right\rangle \\
& =\int \mathrm{d} z_{\mathrm{c}}\left\langle z_{\mathrm{b}}\left|U\left(t_{\mathrm{b}}, t_{\mathrm{c}}\right)\right| z_{\mathrm{c}}\right\rangle\left\langle z_{\mathrm{c}}\left|U\left(t_{\mathrm{c}}, t_{\mathrm{a}}\right)\right| z_{\mathrm{a}}\right\rangle \\
& =\int \mathrm{d} z_{\mathrm{c}} K\left(z_{\mathrm{b}} t_{\mathrm{b}}, z_{\mathrm{c}} t_{\mathrm{c}}\right) K\left(z_{\mathrm{c}} t_{\mathrm{c}}, z_{\mathrm{a}} t_{\mathrm{a}}\right) .
\end{aligned}
$$

The last line is an expression of the composition property of the quantum propagator, and may be interpreted as a summation over intermediate states. All possible paths connecting the initial spacetime point $z_{\mathrm{a}} t_{\mathrm{a}}$ to the final spacetime point $z_{\mathrm{b}} t_{\mathrm{b}}$ must pass through some position $z_{\mathrm{c}}$ at the intermediate time $t_{c}$ (see Fig. 3 ). The amplitude for passing through this particular intermediate state is just the product $K\left(z_{\mathrm{b}} t_{\mathrm{b}}, z_{\mathrm{c}} t_{\mathrm{c}}\right) K\left(z_{\mathrm{c}} t_{\mathrm{c}}, z_{\mathrm{a}} t_{\mathrm{a}}\right)$. The total amplitude $K\left(z_{\mathrm{b}} t_{\mathrm{b}}, z_{\mathrm{a}} t_{\mathrm{a}}\right)$ for arriving at the final point $z_{\mathrm{b}} t_{\mathrm{b}}$ from the initial point $z_{\mathrm{a}} t_{\mathrm{a}}$ equals the sum of the amplitudes for passing through all possible intermediate positions $z_{\mathrm{c}}$ at time $t_{\mathrm{c}}$. The fact that this composition rule applies to amplitudes rather than probabilities is a distinguishing feature of quantum mechanics.

1.2.3 Feynman's expression for the quantum propagator. - The composition property is the starting point of Feynman's formulation, which defines the quantum propagator as a sum of 


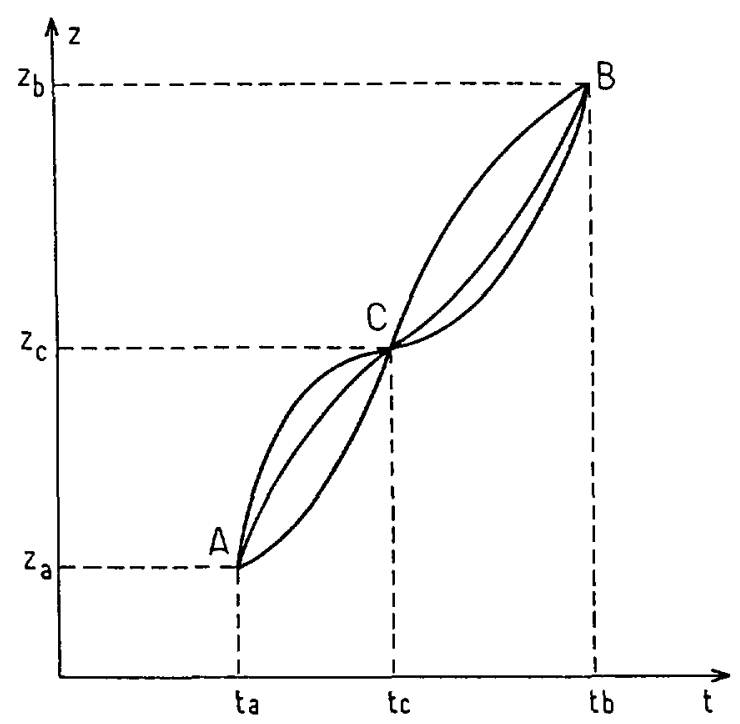

Fig. 3. - Possible paths connecting points $z_{\mathrm{a}} t_{\mathrm{a}}$ and $z_{\mathrm{b}} t_{\mathrm{b}}$ which pass through the intermediate point $z_{\mathrm{c}} t_{\mathrm{c}}$.

contributions from all possible paths connecting the initial and final points $[4,5]$. The modulus of each contribution is independent of the path $\Gamma$. However the phase factor equals $S_{\Gamma} / \hbar$, where $S_{\Gamma}$ is the action along the path $\Gamma$. Feynman's expression for the quantum propagator may be written as

$$
K\left(z_{\mathrm{b}} t_{\mathrm{b}}, z_{\mathrm{a}} t_{\mathrm{a}}\right)=\mathcal{N} \sum_{\Gamma} \mathrm{e}^{2 S_{\Gamma} / \hbar}
$$

where $\mathcal{N}$ is a normalization constant, and $\sum_{\Gamma}$ denotes a functional integral over the space of all possible paths $\Gamma$ connecting $z_{\mathrm{a}} t_{\mathrm{a}}$ and $z_{\mathrm{b}} t_{\mathrm{b}}$. An alternative notation is

$$
K\left(z_{\mathrm{b}} t_{\mathrm{b}}, z_{\mathrm{a}} t_{\mathrm{a}}\right) \equiv K(b, a)=\int_{\mathrm{a}}^{b} \mathcal{D} z(t) \mathrm{e}^{2 S_{\Gamma} / \hbar},
$$

where $\mathcal{D} z(t)$ denotes the element of integration. It can be shown $[4,5]$ that Feynman's expression is completely equivalent to the usual definition (16).

In the classical limit where $S_{\Gamma} \gg \hbar$, the phase $S_{\Gamma} / \hbar$ generally varies very rapidly between neighbouring paths $\Gamma$, and destructive interference occurs. Along the classical path however the action is extremal, and constructive interference occurs among the neighbouring paths. Therefore only the paths close to the classical path will contribute to the integral (20). This explains how quantum mechanics reduces to classical mechanics in the limit $\hbar \rightarrow 0$.

1.2.4 The quantum propagator for quadratic Lagrangians. - A simple form can be found for the propagator if the Lagrangian is a quadratic function of $z$ and $\dot{z}$, that is if has the form

$$
L=a(t) \dot{z}^{2}+b(t) \dot{z} z+c(t) z^{2}+d(t) \dot{z}+e(t) z+f(t) .
$$

Three examples of quadratic Lagrangians are those of a free particle,

$$
L=\frac{1}{2} M \dot{z}^{2},
$$


a particle in a gravitational field,

$$
L=\frac{1}{2} M \dot{z}^{2}-M g z
$$

and a particle in a reference frame rotating at angular velocity $\boldsymbol{\Omega}$ with respect to a Galilean frame [3]

$$
L=\frac{1}{2} M \dot{\mathbf{r}}^{2}+M \boldsymbol{\Omega} \cdot(\mathbf{r} \times \dot{\mathbf{r}})+\frac{1}{2} M(\boldsymbol{\Omega} \times \mathbf{r})^{2}
$$

Since only paths very close to the classical path are expected to contribute to the integral (20), it is convenient to express each path $z(t)$ in terms of its deviation from the classical path $\bar{z}(t)$

$$
z(t)=\bar{z}(t)+\xi(t)
$$

where the boundary conditions are

$$
z\left(t_{\mathrm{a}}\right)=\bar{z}\left(t_{\mathrm{a}}\right)=z_{\mathrm{a}}, z\left(t_{\mathrm{b}}\right)=\bar{z}\left(t_{\mathrm{b}}\right)=z_{\mathrm{b}}, \xi\left(t_{\mathrm{a}}\right)=\xi\left(t_{\mathrm{b}}\right)=0 .
$$

In terms of this deviation $\xi(t)$ from the classical path, expression (20) for the quantum propagator can be written as

$$
K\left(z_{\mathrm{b}} t_{\mathrm{b}}, z_{\mathrm{a}} t_{\mathrm{a}}\right)=\int_{0}^{0} \mathcal{D} \xi(t) \exp \left\{\frac{i}{\hbar} S[\bar{z}(t)+\xi(t)]\right\} .
$$

Substituting (25) into (21) gives an expression for the Lagrangian which is quadratic in $\bar{z}, \dot{\bar{z}}, \xi$ and $\dot{\xi}$. We will consider the contribution to the action $S[\bar{z}(t)+\xi(t)]$ from each of the terms in turn. The terms which are independent of $\xi$ and $\dot{\xi}$ contribute the value $S[\bar{z}(t)]$, which is just the classical action $S_{\mathrm{cl}}\left(z_{\mathrm{b}} t_{\mathrm{b}}, z_{\mathrm{a}} t_{\mathrm{a}}\right)$. The contribution from the terms that are linear in $\xi$ and $\dot{\xi}$ is the first-order difference in the action between the path $z(t)$ and the classical path $\bar{z}(t)$. This is zero, since the action is extremal along the classical path. The contribution from the quadratic terms is however nonzero. Writing this term out explicitly, we have

$$
S[\bar{z}(t)+\xi(t)]=S_{\mathrm{cl}}\left(z_{\mathrm{b}} t_{\mathrm{b}}, z_{\mathrm{a}} t_{\mathrm{a}}\right)+\int_{t_{\mathrm{a}}}^{t_{\mathrm{b}}} \mathrm{d} t\left[a(t) \dot{\xi}^{2}+b(t) \dot{\xi} \xi+c(t) \xi^{2}\right] .
$$

Substituting the above expression into equation (27) for the propagator gives

$$
\begin{aligned}
K\left(z_{\mathrm{b}} t_{\mathrm{b}}, z_{\mathrm{a}} t_{\mathrm{a}}\right)= & \exp \left\{\frac{i}{\hbar} S_{\mathrm{cl}}\left(z_{\mathrm{b}} t_{\mathrm{b}}, z_{\mathrm{a}} t_{\mathrm{a}}\right)\right\} \times \\
& \int_{0}^{0} \mathcal{D} \xi(t) \exp \left\{\frac{i}{\hbar} \int_{t_{\mathrm{a}}}^{t_{\mathrm{b}}} \mathrm{d} t\left[a(t) \dot{\xi}^{2}+b(t) \dot{\xi} \xi+c(t) \xi^{2}\right]\right\} .
\end{aligned}
$$

The functional integral in the above equation is independent of $z_{\mathrm{a}}$ and $z_{\mathrm{b}}$, and may be denoted by $F\left(t_{\mathrm{b}}, t_{\mathrm{a}}\right)$. Hence the following simplified expression for the propagator is obtained

$$
K\left(z_{\mathrm{b}} t_{\mathrm{b}}, z_{\mathrm{a}} t_{\mathrm{a}}\right)=F\left(t_{\mathrm{b}}, t_{\mathrm{a}}\right) \exp \left\{\frac{i}{\hbar} S_{\mathrm{cl}}\left(z_{\mathrm{b}} t_{\mathrm{b}}, z_{\mathrm{a}} t_{\mathrm{a}}\right)\right\} .
$$

1.3 Propagation of the wavefunction. - We now use the result (30) to calculate the propagation of the wavefunction in the case where the Lagrangian is quadratic. A simple expression for the final wavefunction is then obtained by assuming that the initial state is a plane wave. The phase shift due to a perturbation on the Lagrangian is evaluated, and the result applied to interferometry calculations. 
1.3.1 Wavefunction calculation for a quadratic Lagrangian. - For a quadratic Lagrangian the propagator is given by expression (30), in which the $z_{\mathrm{a}}$ dependence is entirely contained in the action $S_{\mathrm{cl}}\left(z_{\mathrm{b}} t_{\mathrm{b}}, z_{\mathrm{a}} t_{\mathrm{a}}\right)$. Substituting this expression into equation (15) gives the evolution of the wavefunction

$$
\psi\left(z_{\mathrm{b}}, t_{\mathrm{b}}\right)=F\left(t_{\mathrm{b}}, t_{\mathrm{a}}\right) \int \mathrm{d} z_{\mathrm{a}} \exp \left\{\frac{i}{\hbar} S_{\mathrm{cl}}\left(z_{\mathrm{b}} t_{\mathrm{b}}, z_{\mathrm{a}} t_{\mathrm{a}}\right)\right\} \psi\left(z_{\mathrm{a}}, t_{\mathrm{a}}\right)
$$

In the integral over $z_{\mathrm{a}}$, the neighbourhood of the position where the phases of $\exp \left\{i S_{\mathrm{cl}}\left(z_{\mathrm{b}} t_{\mathrm{b}}\right.\right.$, $\left.\left.z_{\mathrm{a}} t_{\mathrm{a}}\right) / \hbar\right\}$ and $\psi\left(z_{\mathrm{a}}, t_{\mathrm{a}}\right)$ cancel out will be predominant. This position is known as the point of "stationary phase".

1.3.2 Plane wave incident. - In the case where the initial wavefunction is a plane wave

$$
\psi\left(z_{\mathrm{a}}, t_{\mathrm{a}}\right)=\frac{1}{\sqrt{2 \pi \hbar}} \exp \left[\frac{i\left(p_{0} z_{\mathrm{a}}-E_{0} t_{\mathrm{a}}\right)}{\hbar}\right]
$$

the method of stationary phase gives an intuitively appealing result for the final wavefunction. Using equation (13) the action is expanded about the position $z_{0}$ where the initial momentum on the classical path $z_{0} t_{\mathrm{a}} \rightarrow z_{\mathrm{b}} t_{\mathrm{b}}$ is the same as the momentum $p_{0}$ of the incident wave. This position $z_{0}$ will turn out to be the point of stationary phase. Since the Lagrangian is quadratic in the position and velocity, it can be shown that the classical action is a quadratic function of the initial and final positions. This can be verified for all the systems that we consider: the free particle (55), a particle in a gravitational field (73), and a particle in a rotating reference frame (97). The expansion for the classical action about $z_{0}$ therefore terminates after the second order term,

$$
S_{\mathrm{cl}}\left[z_{\mathrm{b}}, t_{\mathrm{b}} ; z_{0}+\zeta, t_{\mathrm{a}}\right]=S_{\mathrm{cl}}\left(z_{\mathrm{b}} t_{\mathrm{b}}, z_{0} t_{\mathrm{a}}\right)-p_{0} \zeta+C\left(t_{\mathrm{b}}, t_{\mathrm{a}}\right) \zeta^{2}
$$

where

$$
\begin{aligned}
p_{0} & =-\frac{\partial}{\partial z_{0}} S_{\mathrm{cl}}\left(z_{\mathrm{b}} t_{\mathrm{b}}, z_{0} t_{\mathrm{a}}\right) \\
C\left(t_{\mathrm{b}}, t_{\mathrm{a}}\right) & \equiv \frac{1}{2} \frac{\partial^{2}}{\partial z_{0}^{2}} S_{\mathrm{cl}}\left(z_{\mathrm{b}} t_{\mathrm{b}}, z_{0} t_{\mathrm{a}}\right)
\end{aligned}
$$

and $C$ is independent of position. Expanding the initial wavefunction about the same point $z_{0}$ gives

$$
\begin{aligned}
\psi\left(z_{0}+\zeta, t_{\mathrm{a}}\right) & =\frac{1}{\sqrt{2 \pi \hbar}} \exp \left\{\frac{i\left[p_{0}\left(z_{0}+\zeta\right)-E_{0} t_{\mathrm{a}}\right]}{\hbar}\right\} \\
& =\frac{1}{\sqrt{2 \pi \hbar}} \exp \left[\frac{\imath\left(p_{0} z_{0}-E_{0} t_{\mathrm{a}}\right)}{\hbar}\right] \exp \left(\frac{i p_{0} \zeta}{\hbar}\right) .
\end{aligned}
$$

When expansions (33) and (36) are substituted into into equation (31), the linear terms in $\zeta$ cancel out in the exponential and one gets

$$
\begin{aligned}
\psi\left(z_{\mathrm{b}}, t_{\mathrm{b}}\right) & =F\left(t_{\mathrm{b}}, t_{\mathrm{a}}\right) \int \mathrm{d} \zeta \exp \left\{\frac{i}{\hbar} S_{\mathrm{cl}}\left[z_{\mathrm{b}}, t_{\mathrm{b}} ; z_{0}+\zeta, t_{\mathrm{a}}\right]\right\} \psi\left(z_{0}+\zeta, t_{\mathrm{a}}\right) \\
& =F\left(t_{\mathrm{b}}, t_{\mathrm{a}}\right) \frac{\mathrm{e}^{2\left(p_{0} z_{0}-E_{0} t_{\mathrm{a}}\right) / \hbar}}{\sqrt{2 \pi \hbar}} \exp \left\{\frac{i}{\hbar} S_{\mathrm{cl}}\left[z_{\mathrm{b}} t_{\mathrm{b}}, z_{0} t_{\mathrm{a}}\right]\right\} \int \mathrm{d} \zeta \mathrm{e}^{\imath C \zeta^{2} / \hbar} \\
& =F\left(t_{\mathrm{b}}, t_{\mathrm{a}}\right) \sqrt{\frac{i \pi \hbar}{C\left(t_{\mathrm{b}}, t_{\mathrm{a}}\right)}} \psi\left(z_{0}, t_{\mathrm{a}}\right) \exp \left\{\frac{i}{\hbar} S_{\mathrm{cl}}\left[z_{\mathrm{b}} t_{\mathrm{b}}, z_{0} t_{\mathrm{a}}\right]\right\}
\end{aligned}
$$


This result has a very simple interpretation. To calculate the final wavefunction at a particular position we consider the trajectory of a classical particle whose initial momentum is $p_{0}$ and which passes through that final point. The phase of the final wavefunction is determined by the action along this classical path, and the phase of the wavefunction at the trajectory's initial point. The amplitude of the final wavefunction is independent of position, and depends only on the initial and final times.

The formalism may be extended to non-quadratic potentials provided the terms of higher than second order are sufficiently small.

1.3.3 Generalizations. - In the above analysis we calculated the value of the wavefunction at a final point $z_{\mathrm{b}} t_{\mathrm{b}}$ by considering the trajectory of a classical particle which arrives at that point. We assumed that it was possible to determine the position $z_{0}$ from which the particle must classically have left, given that it had an initial momentum of $p_{0}$. It may happen that this stationary phase point $z_{0}$ is not unique. In fact if it is not unique, then all initial positions are points of stationary phase. This may be seen as follows. Since the classical action is quadratic in the initial position $z_{\mathrm{a}}$, the momentum is a linear function of $z_{\mathrm{a}}$

$$
p_{\mathrm{a}}=-\frac{\partial S_{\mathrm{cl}}}{\partial z_{\mathrm{a}}}=p_{0}-2 C\left(t_{\mathrm{b}}, t_{\mathrm{a}}\right)\left(z_{\mathrm{a}}-z_{0}\right) .
$$

If at any time $C\left(t_{\mathrm{b}}, t_{\mathrm{a}}\right)$ becomes zero, then the condition $p_{\mathrm{a}}=p_{0}$ is satisfied for all initial positions $z_{\mathrm{a}}$. In this case focussing occurs, and the wavefunction collapses to a delta function. Mathematically, the vanishing of $C\left(t_{b}, t_{a}\right)$ causes a divergence in equation (37).

One system in which this happens is the harmonic oscillator. Consider the set of classical trajectories starting at time $t_{\mathrm{a}}$ with momentum $p_{0}=0$ from all possible initial positions. After a quarter of a period $\tau / 4$ all the trajectories will focus onto the point $z_{\mathrm{b}}=0$. Quantum mechanically, the system starts in a momentum eigenstate, and a quarter of a period later finishes in a position eigenstate. It can be shown for the harmonic oscillator that $C\left[t_{\mathrm{a}}+\right.$ $\left.\tau(1 / 4+m / 2), t_{\mathrm{a}}\right]=0$ for all $t_{\mathrm{a}}$ and all integers $m$.

If the Lagrangian is not quadratic the situation is more complicated since in general both $F$ and $C$ are position dependent. As long as $F$ varies sufficiently slowly with initial position, the stationary phase method may still be used. However two points should be noted. Firstly, there may exist multiple discrete points of stationary phase. In this case the final wavefunction consists of a quantum superposition of solutions, and equation (37) must be replaced by a sum of contributions from each stationary phase point $z_{0}$. Secondly, $C$ may vanish. But since $C$ is position dependent this will occur locally, rather than globally as was the case for quadratic Lagrangians. In general $C$ will vanish only at discrete values of the initial position. In this case the family of classical trajectories will not all converge to a single focal point as described above, but will instead form a caustic. In the neighbourhood of such points the stationary phase method is no longer valid, and the expansion (33) for the action must be extended to include higher order terms. The occurrence of caustics is a very general phenomenon in physics, and is treated by catastrophe theory [6]. More elaborate semi-classical calculations may be performed using the uniform approximation [7].

1.3.4 Perturbative limit. - In this section we discuss the effect of a perturbation on the Lagrangian. We show that the phase shift introduced by the perturbation may be calculated by integrating the perturbation along the unperturbed path.

We consider the same situation as was discussed in section 1.3 .2 , in which the initial wavefunction was a plane wave with momentum $p_{0}$. In that section we calculated the final wavefunction (37) at the point $z_{\mathrm{b}} t_{\mathrm{b}}$ by following the trajectory of a classical particle which had initial momentum $p_{0}$ and which passed through the final point. In preparation for the perturbative 


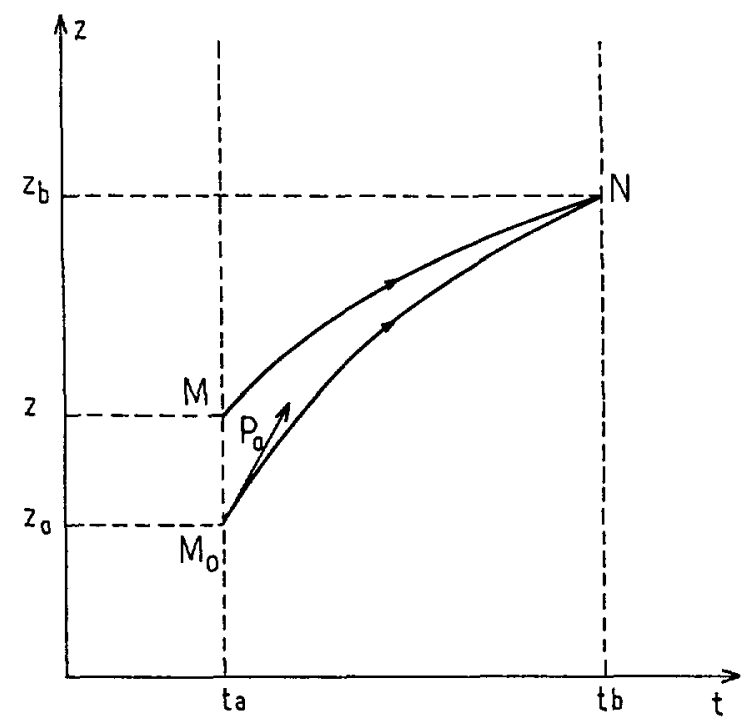

Fig. 4. - Classical paths from two points in the same Fresnel zone.

treatment that follows, we show first that this trajectory can be replaced by a neighbouring classical trajectory which passes through the same final point. This replacement is possible provided the initial positions of the two trajectories are sufficiently close.

Consider the two classical paths sketched in figure 4. Both trajectories pass through the same final point $z_{\mathrm{b}} t_{\mathrm{b}}$, labeled $N$. The trajectory which departs from the point $M_{0}$ has initial momentum $p_{0}$, and corresponds to the point $z_{0} t_{\mathrm{a}}$ of stationary phase which was used in section 1.3.2 to calculate the final wavefunction. The point $M$ is a neighbouring point with coordinates $z t_{\mathrm{a}}$.

Using equation (37) we obtain for the final wavefunction (up to an amplitude factor)

$$
\psi(\mathrm{N})=\psi\left(\mathrm{M}_{0}\right) \exp \left\{i S_{\mathrm{cl}}\left(\mathrm{N}, \mathrm{M}_{0}\right) / \hbar\right\}
$$

where $S_{\mathrm{cl}}\left(\mathrm{N}, \mathrm{M}_{0}\right)$ is the action along the classical trajectory connecting the points $\mathrm{M}_{0}$ and $\mathrm{N}$.

We now consider the neighbouring point $M$. Along the trajectory connecting $M$ to $N$ we have

$$
\begin{aligned}
& \psi(\mathrm{M}) \exp \left\{i S_{\mathrm{cl}}(\mathrm{N}, \mathrm{M}) / \hbar\right\} \\
= & \frac{\mathrm{e}^{2\left(p_{0} z-E_{0} t_{\mathrm{a}}\right) / \hbar}}{\sqrt{2 \pi \hbar}} \exp \left\{\frac{i}{\hbar}\left[S_{\mathrm{cl}}\left(z_{\mathrm{b}} t_{\mathrm{b}}, z_{0} t_{\mathrm{a}}\right)-p_{0}\left(z-z_{0}\right)+C\left(z-z_{0}\right)^{2}\right]\right\} \\
= & \frac{\mathrm{e}^{2\left(p_{0} z_{0}-E_{0} t_{\mathrm{a}}\right) / \hbar}}{\sqrt{2 \pi \hbar}} \exp \left\{\frac{i}{\hbar}\left[S_{\mathrm{cl}}\left(z_{\mathrm{b}} t_{\mathrm{b}}, z_{0} t_{\mathrm{a}}\right)+C\left(z-z_{0}\right)^{2}\right]\right\} \\
= & \psi\left(\mathrm{M}_{0}\right) \exp \left\{i S_{\mathrm{cl}}\left(\mathrm{N}, \mathrm{M}_{0}\right) / \hbar\right\} \exp \left\{\frac{i}{\hbar} C\left(z-z_{0}\right)^{2}\right\} .
\end{aligned}
$$

Hence we can make the approximation

$$
\psi\left(\mathrm{M}_{0}\right) \exp \left\{i S_{\mathrm{cl}}\left(\mathrm{N}, \mathrm{M}_{0}\right) / \hbar\right\} \approx \psi(\mathrm{M}) \exp \left\{i S_{\mathrm{cl}}(\mathrm{N}, \mathrm{M}) / \hbar\right\}
$$

if the point $M$ is sufficiently close to $M_{0}$ that the final exponential factor of equation (40) can 


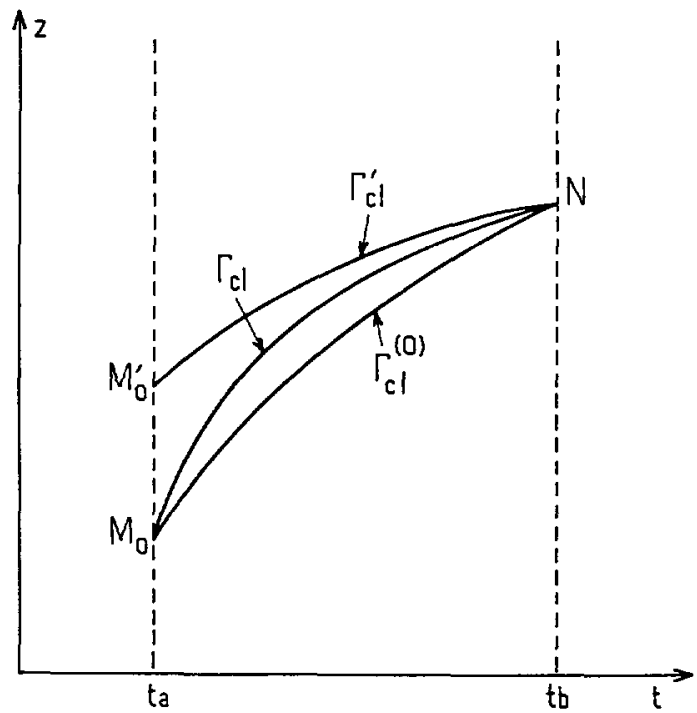

Fig. 5. - Perturbed and unperturbed paths used to calculate the perturbative phase shift.

be replaced by unity. Quantitatively, this condition may be expressed as

$$
\frac{C}{\hbar}\left(\overline{\mathrm{M}_{0} \mathrm{M}}\right)^{2} \ll 1
$$

where $\overline{M_{0}} \bar{M}$ is the distance between the positions $M_{0}$ and $M$. When this is satisfied the points $\mathrm{M}_{0}$ and $\mathrm{M}$ can be said to lie in the same "Fresnel zone". All points in this zone radiate approximately in phase with $\mathrm{M}_{0}$. We will use this approximation in the perturbative calculation of the wavefunction which follows.

We consider a perturbation on the Lagrangian

$$
L=L_{0}+\epsilon L_{1}
$$

where $\epsilon \ll 1$. We wish to calculate the phase shift introduced by this perturbation on the final wavefunction, given that the initial wavefunction is a plane wave with momentum $p_{0}$. In figure $5, \Gamma_{\mathrm{cl}}^{(0)}$ denotes the classical path with initial momentum $p_{0}$ corresponding to the unperturbed Lagrangian $(\epsilon=0)$. Its initial point is labeled $\mathrm{M}_{0} . \Gamma_{\mathrm{cl}}$ is the classical path linking the same initial and final points $\mathrm{M}_{0}$ and $\mathrm{N}$, but corresponding to perturbed Lagrangian $(\epsilon \neq 0)$. (The initial momentum on this path will not in general be equal to $\left.p_{0}.\right) \Gamma_{\mathrm{cl}}^{\prime}$ is the classical path with initial momentum $p_{0}$ corresponding to the perturbed Lagrangian $(\epsilon \neq 0)$. Its initial point is labeled $\mathrm{M}_{0}^{\prime}$. The situation can be summarized as follows

$$
\begin{array}{llll}
\Gamma_{\mathrm{cl}}^{(0)} \cdot & \mathrm{M}_{0} \rightarrow \mathrm{N} & \epsilon=0 & p\left(t_{\mathrm{a}}\right)=p_{0} \\
\Gamma_{\mathrm{cl}}: & \mathrm{M}_{0} \rightarrow \mathrm{N} & \epsilon \neq 0 & p\left(t_{\mathrm{a}}\right) \neq p_{0} \\
\Gamma_{\mathrm{cl}}^{\prime} . & \mathrm{M}_{0}^{\prime} \rightarrow \mathrm{N} & \epsilon \neq 0 & p\left(t_{\mathrm{a}}\right)=p_{0}
\end{array}
$$

The perturbation introduces a phase shift between the unperturbed wavefunction $\psi^{(0)}(\mathrm{N})$ and the perturbed wavefunction $\psi(\mathrm{N})$. Up to amplitude factors these wavefunctions are given 
by

$$
\begin{aligned}
\psi^{(0)}(\mathrm{N}) & =\psi\left(\mathrm{M}_{0}\right) \exp \left\{\frac{i}{\hbar} \int_{\Gamma_{\mathrm{cl}}^{(0)}} L_{0} \mathrm{~d} t\right\} \\
\psi(\mathrm{N}) & =\psi\left(\mathrm{M}_{0}^{\prime}\right) \exp \left\{\frac{i}{\hbar} \int_{\Gamma_{\mathrm{cl}}^{\prime}}\left(L_{0}+\epsilon L_{1}\right) \mathrm{d} t\right\} .
\end{aligned}
$$

If $M_{0}$ and $M_{0}^{\prime}$ are sufficiently close (in the sense of condition (42)), then the perturbed wavefunction can be approximated by

$$
\psi(\mathrm{N}) \approx \psi\left(\mathrm{M}_{0}\right) \exp \left\{\frac{i}{\hbar} \int_{\Gamma_{\mathrm{cl}}}\left(L_{0}+\epsilon L_{1}\right) \mathrm{d} t\right\}
$$

However $\Gamma_{\mathrm{cl}}$ and $\Gamma_{\mathrm{cl}}^{(0)}$ have the same endpoints and differ to first order in $\epsilon$. Since $\Gamma_{\mathrm{cl}}$ is the path which maximizes the action for $\epsilon \neq 0$,

$$
\int_{\Gamma_{\mathrm{cl}}} L \mathrm{~d} t=\int_{\Gamma_{\mathrm{rl}}^{(0)}} L \mathrm{~d} t+O\left(\epsilon^{2}\right)
$$

Substituting this equation into (47) we find that to first order in $\epsilon$

$$
\psi(\mathrm{N}) \approx \psi\left(\mathrm{M}_{0}\right) \exp \left\{\frac{i}{\hbar} \int_{\mathrm{\Gamma}_{\mathrm{cl}}^{(0)}}\left(L_{0}+\epsilon L_{1}\right) \mathrm{d} t\right\} .
$$

Expression (45) then gives

$$
\psi(\mathrm{N}) \approx \psi^{(0)}(\mathrm{N}) \exp \left\{\frac{i}{\hbar} \int_{\Gamma_{\mathrm{c} 1}^{(0)}} \epsilon L_{1} \mathrm{~d} t\right\}=\psi^{(0)}(\mathrm{N}) \mathrm{e}^{\imath \delta \phi},
$$

where

$$
\delta \phi=\frac{\epsilon}{\hbar} \int_{\Gamma_{\mathrm{cl}}^{(0)}} L_{1} \mathrm{~d} t
$$

This result shows that to first order in $\epsilon$, the phase shift $\delta \phi$ introduced into the final wavefunction by the perturbation is determined simply by the integral of the perturbation along the unperturbed path.

1.3.5 Application to interferometry. - Here we calculate the phase shift introduced between the two arms of an interferometer due to a perturbation on the Langrangian.

Figure 6 represents the two possible unperturbed classical paths $\mathrm{A}^{\prime} \mathrm{CB}$ (labeled I) and ADB (labeled II) which can be followed by the particle through the two arms of the interferometer, and which arrive at the same final point $\mathrm{B}$ at $t=t_{\mathrm{b}}$. We have considered here the most general situation, in which the two classical paths start at the initial time from two different points $A^{\prime}$ and $A$. This is the case for example in a Michelson type interferometer where the lengths of the two arms (between the beamsplitter and the two reflecting mirrors) are unequal. Very often however the points $\mathrm{A}^{\prime}$ and $\mathrm{A}$ coincide (see for example Fig. 9).

According to equation (51) the phase shifts induced by the perturbation along the paths marked I and II in the interferometer are

$$
\begin{aligned}
\delta \phi_{\mathrm{I}} & =\frac{\epsilon}{\hbar} \int_{\mathrm{A}^{\prime} \mathrm{CB}} L_{1} \mathrm{~d} t \\
\delta \phi_{\mathrm{II}} & =\frac{\epsilon}{\hbar} \int_{\mathrm{ADB}} L_{1} \mathrm{~d} t .
\end{aligned}
$$




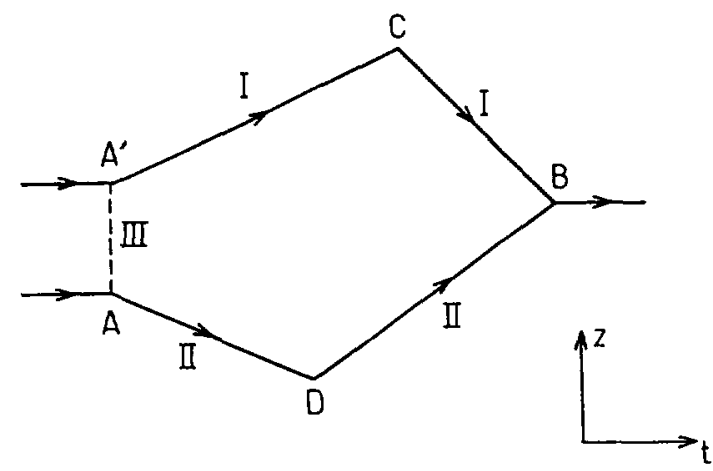

Fig. 6. - Two spacetime paths through an interferometer.

Section III of the diagram (segment $\mathrm{AA}^{\prime}$ ) is not a classical path. However the phase shift between the points $A$ and $A^{\prime}$ is known. It is independent of the perturbation, and equals $p_{0} \Delta x / \hbar$, where $p_{0}$ is the initial momentum and $\Delta x$ is the displacement between the two points.

The total phase shift between the two arms introduced by the perturbation is therefore

$$
\delta \phi=\delta \phi_{\mathrm{I}}-\delta \phi_{\mathrm{II}}=\frac{\epsilon}{\hbar} \oint_{\mathrm{AA}^{\prime} \mathrm{CBDA}} L_{1} \mathrm{~d} t
$$

where we have replaced $\int_{\mathrm{A}^{\prime} \mathrm{CBDA}}$ by $\oint_{\mathrm{AA}^{\prime} \mathrm{CBDA}}$ since $\int_{\mathrm{A}^{\prime} \mathrm{A}} L_{1} \mathrm{~d} t=0$.

This final result shows that the phase shift introduced by a perturbation on the Lagrangian is determined simply by the integral of the perturbation around the closed unperturbed path comprising the two arms of the interferometer.

\section{Examples in interferometry.}

In this part we apply the formalism developed above to several systems of current interest. We first consider the propagation of a free particle (Sect. 2.1) and the modification of the wavefunction of a 2-level atom crossing a laser wave (Sect. 2.2), since several atomic interferometers consist of free propagation zones separated by laser-atom interactions. The results of sections 2.1 and 2.2 will be used in the analysis of the experiment of Kasevich and Chu in section 2.3 .

2.1 The fREe PARTICLE. - The Lagrangian for a free particle contains just the kinetic energy term,

$$
L=\frac{1}{2} M \dot{z}^{2} .
$$

Since the velocity remains constant, the classical paths are simply straight lines in space-time. The classical action is therefore

$$
S_{\mathrm{cl}}\left(z_{\mathrm{b}} t_{\mathrm{b}}, z_{\mathrm{a}} t_{\mathrm{a}}\right)=\int_{t_{\mathrm{a}}}^{t_{\mathrm{b}}} \mathrm{d} t \frac{1}{2} M \dot{z}^{2}=\frac{M}{2} \frac{\left(z_{\mathrm{b}}-z_{\mathrm{a}}\right)^{2}}{t_{\mathrm{b}}-t_{\mathrm{a}}}
$$

The quantum propagator can be calculated directly using an expansion in terms of momentum states, which are eigenstates of the evolution operator,

$$
\left\langle z_{\mathrm{b}}\left|U\left(t_{\mathrm{b}}, t_{\mathrm{a}}\right)\right| z_{\mathrm{a}}\right\rangle=\int \mathrm{d} p\left\langle z_{\mathrm{b}} \mid p\right\rangle \exp \left[-i \frac{p^{2}\left(t_{\mathrm{b}}-t_{\mathrm{a}}\right)}{2 M \hbar}\right]\left\langle p \mid z_{\mathrm{a}}\right\rangle
$$




$$
\begin{aligned}
& =\frac{1}{2 \pi \hbar} \int \mathrm{d} p \exp \left\{-i\left[\frac{p^{2}\left(t_{\mathrm{b}}-t_{\mathrm{a}}\right)}{2 M \hbar}+\frac{p\left(z_{\mathrm{a}}-z_{\mathrm{b}}\right)}{\hbar}\right]\right\} \\
& =\sqrt{\frac{M}{i 2 \pi \hbar\left(t_{\mathrm{b}}-t_{\mathrm{a}}\right)}} \exp \left[\frac{i M\left(z_{\mathrm{a}}-z_{\mathrm{b}}\right)^{2}}{2 \hbar\left(t_{\mathrm{b}}-t_{\mathrm{a}}\right)}\right] .
\end{aligned}
$$

Comparing with equation (30), we note that the exponential factor above is indeed $\exp \left(i S_{\mathrm{cl}} / \hbar\right)$, and that the factor $F$ is independent of position

$$
F\left(t_{\mathrm{b}}, t_{\mathrm{a}}\right)=\sqrt{\frac{M}{i 2 \pi \hbar\left(t_{\mathrm{b}}-t_{\mathrm{a}}\right)}}
$$

Evaluating the momentum using expression (6) gives the expected result

$$
p_{\mathrm{a}}=-\frac{\partial}{\partial z_{\mathrm{a}}} S_{\mathrm{cl}}\left(z_{\mathrm{b}} t_{\mathrm{b}}, z_{\mathrm{a}} t_{\mathrm{a}}\right)=M \frac{z_{\mathrm{b}}-z_{\mathrm{a}}}{t_{\mathrm{b}}-t_{\mathrm{a}}}=M v_{\mathrm{a}}
$$

Similarly the Hamiltonian can be calculated using expression (9), which yields

$$
H_{\mathrm{a}}=\frac{\partial}{\partial t_{\mathrm{a}}} S_{\mathrm{cl}}\left(z_{\mathrm{b}} t_{\mathrm{b}}, z_{\mathrm{a}} t_{\mathrm{a}}\right)=\frac{M}{2}\left(\frac{z_{\mathrm{b}}-z_{\mathrm{a}}}{t_{\mathrm{b}}-t_{\mathrm{a}}}\right)^{2}=\frac{1}{2} M v_{\mathrm{a}}^{2}
$$

Definition (35) for $C$ gives

$$
C\left(t_{\mathrm{b}}, t_{\mathrm{a}}\right)=\frac{1}{2} \frac{\partial^{2}}{\partial z_{\mathrm{a}}^{2}} S_{\mathrm{cl}}\left(z_{\mathrm{b}} t_{\mathrm{b}}, z_{\mathrm{a}} t_{\mathrm{a}}\right)=-\frac{1}{2} \frac{\partial p_{\mathrm{a}}}{\partial z_{\mathrm{a}}}=\frac{M}{2\left(t_{\mathrm{b}}-t_{\mathrm{a}}\right)} .
$$

The propagation of a plane wave with initial momentum $p_{0}$ can be calculated using equation (37). The value of the wavefunction at the initial point is

$$
\psi\left(z_{0}, t_{\mathrm{a}}\right)=\frac{1}{\sqrt{2 \pi \hbar}} \exp \left[\frac{i}{\hbar}\left(p_{0} z_{0}-E_{0} t_{\mathrm{a}}\right)\right]
$$

where $E_{0}=p_{0}^{2} / 2 M$. The phase $\phi\left(z_{\mathrm{b}}, t_{\mathrm{b}}\right)$ of the wavefunction at the final point is therefore

$$
\begin{aligned}
\phi\left(z_{\mathrm{b}}, t_{\mathrm{b}}\right) & =\frac{p_{0} z_{0}-E_{0} t_{\mathrm{a}}}{\hbar}+\frac{S_{\mathrm{cl}}\left(z_{\mathrm{b}} t_{\mathrm{b}}, z_{0} t_{\mathrm{a}}\right)}{\hbar} \\
& =\frac{2 M p_{0} z_{0}-p_{0}^{2} t_{\mathrm{a}}}{2 M \hbar}+\frac{p_{0}^{2}\left(t_{\mathrm{b}}-t_{\mathrm{a}}\right)}{2 M \hbar} \\
& =\frac{p_{0} z_{\mathrm{b}}-E_{0} t_{\mathrm{b}}}{\hbar},
\end{aligned}
$$

since $z_{\mathrm{b}}-z_{0}=p_{0}\left(t_{\mathrm{b}}-t_{\mathrm{a}}\right) / M$. Using equations (57) and (60) we obtain the amplitude $A\left(t_{\mathrm{b}}\right)$ of the wavefunction at the final point

$$
A\left(t_{\mathrm{b}}\right)=F\left(t_{\mathrm{b}}, t_{\mathrm{a}}\right) \sqrt{\frac{i \pi \hbar}{C\left(t_{\mathrm{b}}, t_{\mathrm{a}}\right)}} \frac{1}{\sqrt{2 \pi \hbar}}=\frac{1}{\sqrt{2 \pi \hbar}} .
$$

Hence the wavefunction at the final point is, as expected,

$$
\psi\left(z_{\mathrm{b}}, t_{\mathrm{b}}\right)=A\left(t_{\mathrm{b}}\right) \mathrm{e}^{\imath \phi\left(z_{\mathrm{b}}, t_{\mathrm{b}}\right)}=\frac{1}{\sqrt{2 \pi \hbar}} \exp \left[\frac{i}{\hbar}\left(p_{0} z_{\mathrm{b}}-E_{0} t_{\mathrm{b}}\right)\right] .
$$


a)

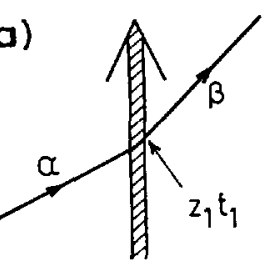

$U_{\beta \alpha} e^{i\left(k_{L} z_{1}-\omega_{L} t_{1}-\varphi\right)}$

c)

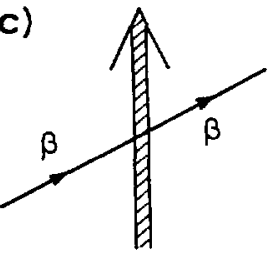

$U_{\beta \beta}$

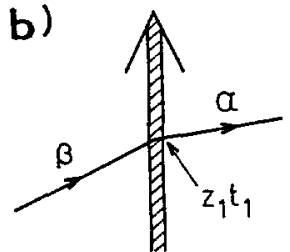

$U_{\alpha \beta} e^{-i\left(k_{L} z_{1}-\omega_{L} L_{1}-\varphi\right)}$

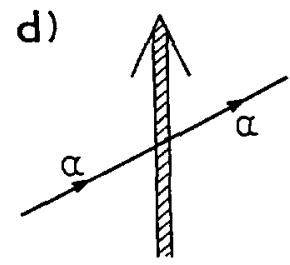

$U_{\alpha \alpha}$

Fig. 7. - Four possible interaction processes involving a two-level atom and a travelling laser wave: (a) atom absorbs a photon, gaining momentum $\hbar k_{\mathrm{L}}$, and making a transition from state $\alpha$ to state $\beta$, (b) atom emits a photon, losing momentum $\hbar k_{\mathrm{L}}$, and decaying from state $\beta$ to state $\alpha$, (c) atom remains in state $\beta,(\mathrm{d})$ atom remains in state $\alpha$.

2.2 A 2-level ATOM CRossing a tRavelling laser Wave. - We consider a freelypropagating two-level atom which interacts with a travelling laser wave. Initially the atom is assumed to have a well-defined momentum, and to be in one of the two internal states $\alpha$ or $\beta$. The interaction with the laser field may change the state of the atom, accompanied by a change in transverse momentum, as shown in figure 7 [2]. We assume that the longitudinal velocity of the atom is sufficiently great that the transverse propagation of the atom (along the laser beam) during the crossing can be neglected. Hence we associate with the interaction a single spacetime point $z_{1} t_{1}$. Depending on the initial and final states of the atom, the effect of the laser interaction is to change the atomic wavefunction by one of four multiplying factors:

$$
\begin{array}{ll}
U_{\beta \alpha} \exp \left\{i\left(k_{\mathrm{L}} z_{1}-\omega_{\mathrm{L}} t_{1}-\phi\right)\right\} & (\alpha \rightarrow \beta) \\
U_{\alpha \beta} \exp \left\{-i\left(k_{\mathrm{L}} z_{1}-\omega_{\mathrm{L}} t_{1}-\phi\right)\right\} & (\beta \rightarrow \alpha) \\
U_{\alpha \alpha} & (\alpha \rightarrow \alpha) \\
U_{\beta \beta} & (\beta \rightarrow \beta)
\end{array}
$$

The quantities $k_{\mathrm{L}}, \omega_{\mathrm{L}}$ and $\phi$ denote the wavenumber, frequency and phase respectively of the laser wave. $U_{\imath}$ is defined as the transition amplitude from the $j$ th to the $i$ th internal atomic state, calculated by taking the coordinate origin to be $z_{1} t_{1}$ and the phase $\phi$ to be zero. Note that the factors $\exp \left\{ \pm i k_{\mathrm{L}} z_{1}\right\}$ are associated with a change of $\pm \hbar k_{\mathrm{L}}$ in the transverse momentum of the atom in the cases when a photon is absorbed or emitted.

As an example we calculate the state of the atom after the interaction, assuming it is in the internal state $\alpha$ and at position $z_{0}$ at the initial time $t_{0}$. We suppose that the atom propagates freely until time $t_{1}$, at which moment it interacts with the laser wave at position $z_{1}$ and makes a transition to the state $\beta$. It then propagates freely again until the final time $t_{2}$, when it reaches the position $z_{2}$. At this final time the atomic wavefunction $\psi_{\beta}\left(z_{2}, t_{2}\right)$ is given by a product of four contributions: the initial wavefunction, a phase shift due to the free propagation before the interaction, a factor due to the interaction itself, and another phase shift due to the free 


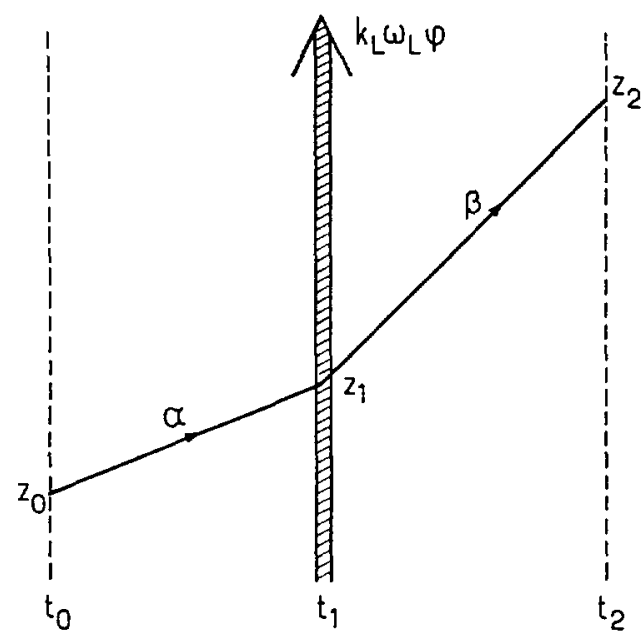

Fig. 8. - The processes of free propagation and photon absorption which determine the final wavefunction $\psi_{\beta}\left(z_{2}, t_{2}\right)$.

propagation after the interaction. These processes are indicated in figure 8 . The result is

$$
\begin{aligned}
\psi_{\beta}\left(z_{2}, t_{2}\right)= & \exp \left\{i S_{\mathrm{cl}}\left(z_{2} t_{2}, z_{1} t_{1}\right) / \hbar\right\} \\
& \times U_{\beta \alpha} \exp \left\{i\left(k_{\mathrm{L}} z_{1}-\omega_{\mathrm{L}} t_{1}-\phi\right)\right\} \\
& \times \exp \left\{i S_{\mathrm{cl}}\left(z_{1} t_{1}, z_{0} t_{0}\right) / \hbar\right\} \psi_{\alpha}\left(z_{0}, t_{0}\right)
\end{aligned}
$$

where $\psi_{\alpha}\left(z_{0}, t_{0}\right)$ is the initial wavefunction of the atom, and where $z_{1}$ and $z_{2}$ are calculated from $z_{0} t_{0}, t_{1}$ and $t_{2}$, knowing the initial momentum $p_{0}$ and the momentum change $\hbar k_{\mathrm{L}}$ due to the laser interaction.

2.3 A particle in a GRAVitational FiEld. - We will now apply the results of the previous two sections to a particle in a gravitational field, and discuss the experiment of Kasevich and Chu [8].

2.3.1 Classical action. - The Lagrangian for a particle in a gravitational field is

$$
L(z, \dot{z})=\frac{1}{2} M \dot{z}^{2}-M g z
$$

Since this is quadratic in $z$ and $\dot{z}$, the results of the previous sections may be directly applied.

The classical paths, which can be derived from the Lagrange equations, are

$$
\begin{aligned}
& v(t)=v_{\mathrm{a}}-g\left(t-t_{\mathrm{a}}\right) \\
& z(t)=z_{\mathrm{a}}+v_{\mathrm{a}}\left(t-t_{\mathrm{a}}\right)-\frac{1}{2} g\left(t-t_{\mathrm{a}}\right)^{2}
\end{aligned}
$$

Evaluating the velocity and position at the final time $t_{\mathrm{b}}$ gives

$$
\begin{aligned}
& v_{\mathrm{b}}=v_{\mathrm{a}}-g\left(t_{\mathrm{b}}-t_{\mathrm{a}}\right) \\
& z_{\mathrm{b}}=z_{\mathrm{a}}+v_{\mathrm{a}}\left(t_{\mathrm{b}}-t_{\mathrm{a}}\right)-\frac{1}{2} g\left(t_{\mathrm{b}}-t_{\mathrm{a}}\right)^{2} .
\end{aligned}
$$


Using this last equation, we can express $v_{\mathrm{a}}$ in terms of the coordinates of the endpoints

$$
v_{\mathrm{a}}=\frac{z_{\mathrm{b}}-z_{\mathrm{a}}}{t_{\mathrm{b}}-t_{\mathrm{a}}}+\frac{1}{2} g\left(t_{\mathrm{b}}-t_{\mathrm{a}}\right)
$$

The action along the classical path is given by

$$
\begin{aligned}
S_{\mathrm{cl}}\left(z_{\mathrm{b}} t_{\mathrm{b}}, z_{\mathrm{a}} t_{\mathrm{a}}\right) & =\int_{t_{\mathrm{a}}}^{t_{\mathrm{b}}} \mathrm{d} t\left[\frac{1}{2} M v(t)^{2}-M g z(t)\right] \\
& =\frac{M}{2} \frac{\left(z_{\mathrm{b}}-z_{\mathrm{a}}\right)^{2}}{t_{\mathrm{b}}-t_{\mathrm{a}}}-\frac{M g}{2}\left(z_{\mathrm{b}}+z_{\mathrm{a}}\right)\left(t_{\mathrm{b}}-t_{\mathrm{a}}\right)-\frac{M g^{2}}{24}\left(t_{\mathrm{b}}-t_{\mathrm{a}}\right)^{3}
\end{aligned}
$$

We note that expression (6) yields the expected result for the momentum

$$
p_{\mathrm{a}}=-\frac{\partial}{\partial z_{\mathrm{a}}} S_{\mathrm{cl}}\left(z_{\mathrm{b}} t_{\mathrm{b}}, z_{\mathrm{a}} t_{\mathrm{a}}\right)=M \frac{z_{\mathrm{b}}-z_{\mathrm{a}}}{t_{\mathrm{b}}-t_{\mathrm{a}}}+\frac{M g}{2}\left(t_{\mathrm{b}}-t_{\mathrm{a}}\right)=M v_{\mathrm{a}}
$$

and expression (9) the expected value of the Hamiltonian

$$
H_{\mathrm{a}}=\frac{\partial}{\partial t_{\mathrm{a}}} S_{\mathrm{cl}}\left(z_{\mathrm{b}} t_{\mathrm{b}}, z_{\mathrm{a}} t_{\mathrm{a}}\right)=\frac{1}{2} M v_{\mathrm{a}}^{2}+M g z_{\mathrm{a}}
$$

2.3.2 The experiment of Kasevich and Chu. - Kasevich and Chu [8] have recently observed atomic interference effects which are sensitive to the gravitational field. They use stimulated Raman transitions between the two hyperfine levels $g_{1}$ and $g_{2}$ of the ground state of sodium to separate an atomic wavepacket into two coherent components and subsequently recombine them. Three Raman pulses are applied using two vertically-oriented counterpropagating laser beams, whose wavenumbers, frequencies and phases are denoted by $k_{1}, \omega_{1}, \phi_{1}$ and $k_{2}, \omega_{2}, \phi_{2}$ respectively (see insert of Fig. 9).

The first pulse (at $t=0$ ) is a $\pi / 2$ pulse, which separates the wavepacket into two components differing by momentum $\hbar\left(k_{1}-k_{2}\right)$. At $t=T$ a $\pi$ pulse is applied, which exchanges the momenta and internal states of the two components. At $t=2 T$, when the two components spatially overlap, another $\pi / 2$ pulse is used to recombine them coherently.

The spacetime paths followed by the atoms are shown in figure 9 . The classical paths in the absence of gravity are straight lines, depicted by the trajectories $A_{0} C_{0} B_{0}$ and $A_{0} D_{0} B_{0}$. In the presence of gravity the trajectories are the parabolic curves represented by ACB and ADB. Note that

$$
\begin{aligned}
& \overline{\mathrm{C}_{0} \mathrm{C}}=\overline{\mathrm{D}_{0} \mathrm{D}}=-\frac{1}{2} g T^{2} \\
& \overline{\mathrm{B}_{0} \mathrm{~B}}=-2 g T^{2} .
\end{aligned}
$$

The gravitational field introduces a phase shift between the interfering beams. We first present an exact calculation of this phase shift using the perturbed trajectories.

From equation (73) the difference between the actions along the two path segments $\mathrm{AC}$ and $\mathrm{AD}$ is

$$
\begin{aligned}
S_{\mathrm{cl}}(\mathrm{AC})-S_{\mathrm{cl}}(\mathrm{AD}) & =\frac{M}{2 T}\left\{\left(z_{\mathrm{C}}-z_{\mathrm{A}}\right)^{2}-\left(z_{\mathrm{D}}-z_{\mathrm{A}}\right)^{2}-g T^{2}\left[\left(z_{\mathrm{C}}+z_{\mathrm{A}}\right)-\left(z_{\mathrm{D}}+z_{\mathrm{A}}\right)\right]\right\} \\
& =\frac{M}{2 T}\left(z_{\mathrm{C}}-z_{\mathrm{D}}\right)\left(z_{\mathrm{C}}+z_{\mathrm{D}}-2 z_{\mathrm{A}}-g T^{2}\right)
\end{aligned}
$$




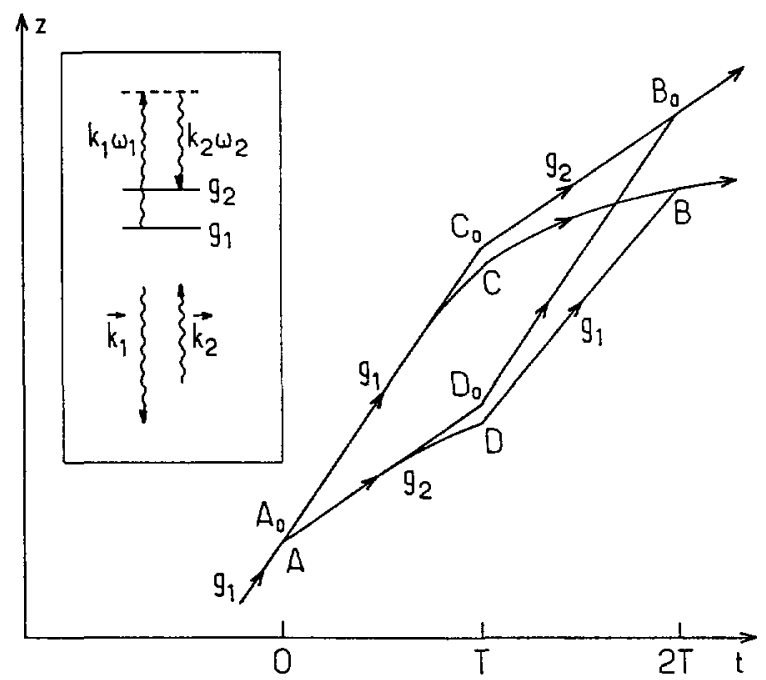

Fig. 9. - Spacetime paths followed by the atoms in the experiment of Kasevich and Chu. Raman pulses occur at times $0, T$ and $2 T$. The insert shows the atomic level scheme and the directions of the laser beams.

Similarly the difference between the actions along the segments CB and DB is

$$
S_{\mathrm{cl}}(\mathrm{CB})-S_{\mathrm{cl}}(\mathrm{DB})=\frac{M}{2 T}\left(z_{\mathrm{C}}-z_{\mathrm{D}}\right)\left(z_{\mathrm{C}}+z_{\mathrm{D}}-2 z_{\mathrm{B}}-g T^{2}\right)
$$

Hence the total contribution to the phase difference between the two arms due to the propagation is

$$
\begin{aligned}
\delta \phi^{\mathrm{prop}} & =S_{\mathrm{cl}}(\mathrm{AC})+S_{\mathrm{cl}}(\mathrm{CB})-\left[S_{\mathrm{cl}}(\mathrm{AD})+S_{\mathrm{cl}}(\mathrm{DB})\right] \\
& =\frac{M}{T}\left(z_{\mathrm{C}}-z_{\mathrm{D}}\right)\left(z_{\mathrm{C}}+z_{\mathrm{D}}-z_{\mathrm{A}}-z_{\mathrm{B}}-g T^{2}\right)
\end{aligned}
$$

However, taking into account relations (76), the second factor in the above expression is

$$
z_{\mathrm{C}}+z_{\mathrm{D}}-z_{\mathrm{A}}-z_{\mathrm{B}}-g T^{2}=z_{\mathrm{C}_{0}}+z_{\mathrm{D}_{0}}-z_{\mathrm{A}_{0}}-z_{\mathrm{B}_{0}}=0
$$

since $A_{0} B_{0} C_{0} D_{0}$ is a parallelogram. Hence

$$
\delta \phi^{\text {prop }}=0 .
$$

Neither is there any contribution $\delta \phi^{\text {int }}$ to the phase difference from the internal evolution, since both components of the wavepacket spend the same amount of time in the two internal states.

We now consider the contribution to the phase difference from the laser interactions. The results of section 2.2 can be directly applied to the Raman pulses after the replacements

$$
\kappa=k_{1}-k_{2}, \omega=\omega_{1}-\omega_{2}, \phi=\phi_{1}-\phi_{2} .
$$

Along the path $\mathrm{ACB}$ the contribution from the laser interactions is

$$
U_{g_{2} g_{2}}^{(3)} U_{g_{2} g_{1}}^{(2)} \exp \left\{i\left[\kappa\left(z_{C_{0}}-\frac{1}{2} g T^{2}\right)-\omega T-\phi_{\mathrm{II}}\right]\right\} U_{g_{1} g_{1}}^{(1)}
$$


where $U^{(1)}, U^{(2)}$ and $U^{(3)}$ are the transition amplitude matrices at times $t=0, t=T$ and $t=2 T$ respectively, and $\phi_{\mathrm{I}}, \phi_{\mathrm{II}}$ and $\phi_{\mathrm{III}}$ are the values of the phase $\phi=\phi_{1}-\phi_{2}$ at these times. Similarly along the path $\mathrm{ADB}$ the contribution from the laser interactions is

$$
\begin{aligned}
& U_{g_{2} g_{1}}^{(3)} \exp \left\{i\left[\kappa\left(z_{\mathrm{B}_{0}}-2 g T^{2}\right)-2 \omega T-\phi_{\mathrm{III}}\right]\right\} \times \\
& U_{g_{1} g_{2}}^{(2)} \exp \left\{-i\left[\kappa\left(z_{\mathrm{D}_{0}}-\frac{1}{2} g T^{2}\right)-\omega T-\phi_{\mathrm{II}}\right]\right\} \times \\
& U_{g_{2} g_{1}}^{(1)} \exp \left\{i\left[\kappa z_{\mathrm{A}_{0}}-\omega \times 0-\phi_{\mathrm{I}}\right]\right\} .
\end{aligned}
$$

Hence the total phase difference between the two arms of the interferometer is

$$
\delta \phi^{\text {tot }}=\delta \phi^{\text {laser }}=\kappa g T^{2}+\phi_{\mathrm{I}}-2 \phi_{\mathrm{II}}+\phi_{\mathrm{III}}
$$

which is sensitive to the gravitational acceleration $g$. (We have neglected the contribution to $\delta \phi^{\text {laser }}$ from the phases of the transition amplitudes $U$, since they are independent of $g$ and of the phases of the lasers.)

We now compare this result with a perturbative calculation of the phase difference, treating the gravitational potential as the perturbation. As described in section 1.3.5 the phase shift can be calculated by integrating the perturbation along the unperturbed paths. The contribution to the phase difference from the laser interactions in this case is obtained from equation (85) by setting $g=0$,

$$
\delta \phi_{0}^{\text {laser }}=\phi_{\mathrm{I}}-2 \phi_{\mathrm{II}}+\phi_{\mathrm{III}} \text {. }
$$

The contribution from the propagation is calculated using equation (53)

$$
\delta \phi_{0}^{\mathrm{prop}}=-\frac{M g}{\hbar} \oint_{\mathrm{A}_{0} \mathrm{C}_{0} \mathrm{~B}_{0} \mathrm{D}_{0} \mathrm{~A}_{0}} \mathrm{~d} t z(t)=-\frac{M g}{\hbar} \mathcal{A},
$$

where $\mathcal{A}$ is the area enclosed by the arms of the interferometer. Using the fact that the distance from the point $\mathrm{D}_{0}$ to $\mathrm{C}_{0}$ is

$$
\overline{\mathrm{D}_{0} \mathrm{C}_{0}}=-\frac{\hbar \kappa}{M} T
$$

we find that the area of the interferometer is

$$
\mathcal{A}=\frac{1}{2} \times 2 T \times \overline{\mathrm{D}_{0} \mathrm{C}_{0}}=-\frac{\hbar \kappa}{M} T^{2},
$$

and the phase shift due to the propagation is

$$
\delta \phi_{0}^{\text {prop }}=\kappa g T^{2}
$$

Since there is no contribution $\delta \phi_{0}^{\text {int }}$ from the internal evolution, the total phase difference between the two arms of the interferometer is

$$
\delta \phi_{0}^{\text {tot }}=\delta \phi_{0}^{\text {prop }}+\delta \phi_{0}^{\text {laser }}=\kappa g T^{2}+\phi_{\mathrm{I}}-2 \phi_{\mathrm{II}}+\phi_{\mathrm{III}}
$$

which agrees with the exact calculation (85).

Note that the phase difference is independent of the initial velocity, and hence remains unchanged after averaging over the velocity spread of the atomic beam. The experiment provides a very precise measurement of the gravitational field, a sensitivity of $\Delta g / g \approx 3 \times 10^{-8}$ being estimated by the authors Kasevich and Chu. 


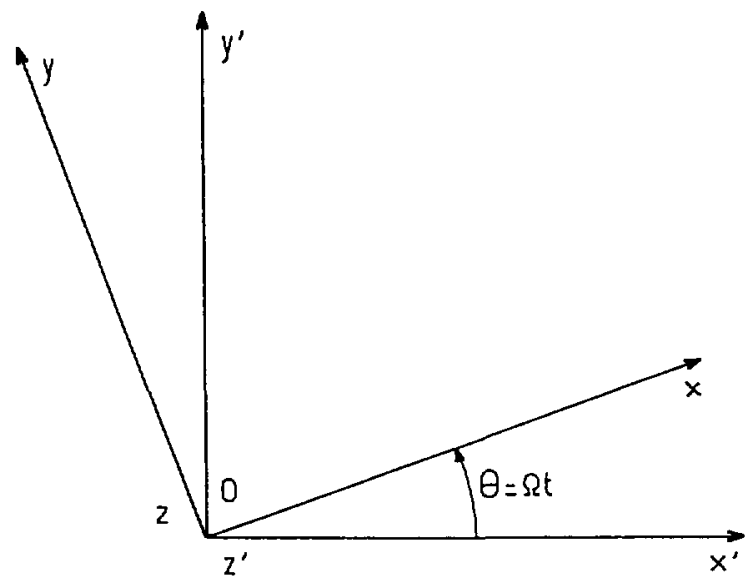

Fig. 10. - Galilean and rotating reference frames.

2.4 A PARTICle in A ROTATING FRAME. - In this section we present a perturbative path integral treatment of a particle in a rotating frame, and apply the results to interferometry. The sensitivity to rotation of matter-wave interferometers is compared with that of optical interferometers (the Sagnac effect).

2.4.1 Classical action. - We define a Galilean frame $\mathcal{R}^{\prime}$ described by the coordinates $x^{\prime} y^{\prime} z^{\prime}$ and a rotating frame $\mathcal{R}$ with coordinates $x y z$, as shown in figure 10. The $z$ axes of the two frames coincide, and frame $\mathcal{R}$ rotates with respect to the Galilean frame $\mathcal{R}^{\prime}$ at angular velocity $\boldsymbol{\Omega}$ where $\boldsymbol{\Omega}=\boldsymbol{\Omega} \mathbf{e}_{z}, \mathbf{e}_{z}$ being the unit vector along the $z$ direction.

The Lagrangian for a free particle, expressed in terms of the coordinates of the Galilean frame $\mathcal{R}^{\prime}$, is

$$
L^{\prime}\left(\mathbf{r}^{\prime}, \mathbf{v}^{\prime}\right)=\frac{1}{2} M \mathbf{v}^{\prime 2}
$$

Since the relation between the velocities $\mathbf{v}^{\prime}$ and $\mathbf{v}$ in the Galilean and rotating frames respectively is

$$
\mathbf{v}^{\prime}=\mathbf{v}+\mathbf{\Omega} \times \mathbf{r},
$$

the Lagrangian can be written in terms of the coordinates of the rotating frame as

$$
\begin{aligned}
L(\mathbf{r}, \mathbf{v}) & =L^{\prime}\left(\mathbf{r}^{\prime}, \mathbf{v}^{\prime}\right)=\frac{1}{2} M(\mathbf{v}+\mathbf{\Omega} \times \mathbf{r})^{2} \\
& =\frac{1}{2} M \mathbf{v}^{2}+M \Omega \cdot(\mathbf{r} \times \mathbf{v})+\frac{1}{2} M(\boldsymbol{\Omega} \times \mathbf{r})^{2}
\end{aligned}
$$

Note that this Lagrangian is quadratic in $\mathbf{r}$ and $\mathbf{v}$.

The momentum is given by

$$
\mathbf{p}=\frac{\partial L}{\partial \mathbf{v}}=M \mathbf{v}+M \boldsymbol{\Omega} \times \mathbf{r}
$$

and the Hamiltonian by

$$
H=\mathbf{p} \cdot \mathbf{v}-L=\frac{\mathbf{p}^{2}}{2 M}-\mathbf{\Omega} \cdot(\mathbf{r} \times \mathbf{p}) .
$$




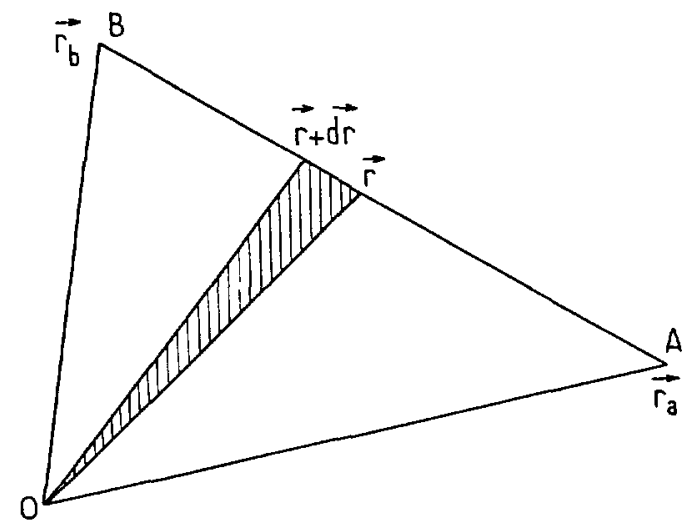

Fig. 11. - Unperturbed path AB in the plane perpendicular to the rotation axis $\boldsymbol{\Omega}$.

A straightforward calculation gives for the classical action

$$
S_{\mathrm{cl}}\left(x_{\mathrm{b}} y_{\mathrm{b}} t_{\mathrm{b}}, x_{\mathrm{a}} y_{\mathrm{a}} t_{\mathrm{a}}\right)=\frac{M}{2\left(t_{\mathrm{b}}-t_{\mathrm{a}}\right)}\left[\left(x_{\mathrm{b}}-x_{\mathrm{a}}\right)^{2}+\left(y_{\mathrm{b}}-y_{\mathrm{a}}\right)^{2}\right]+M \Omega\left(x_{\mathrm{a}} y_{\mathrm{b}}-x_{\mathrm{b}} y_{\mathrm{a}}\right),
$$

which is a quadratic function of $x_{\mathrm{a}}, x_{\mathrm{b}}, y_{\mathrm{a}}$ and $y_{\mathrm{b}}$.

2.4.2 Perturbative calculation of the phase shift. - We now consider the situation in which the angular velocity $\Omega$ and the time intervals $\Delta t$ of interest are sufficiently small that

$$
\Omega \Delta t \ll 1
$$

In this case the rotation can be considered as a perturbation, and the second order terms in $\Omega$ neglected in the Lagrangian

$$
L \approx \frac{1}{2} M \mathbf{v}^{2}+M \Omega \cdot(\mathbf{r} \times \mathbf{v}) .
$$

The phase shift due to the perturbation is calculated along the unperturbed path, which is a straight line segment as shown in figure 11. Note that unlike the previous trajectory diagrams, which were spacetime representations, this figure is in real space, and depicts the plane perpendicular to the rotation axis $\Omega$. The particle leaves position $\mathbf{r}_{\mathrm{a}}$ at time $t_{\mathrm{a}}$ and arrives at position $\mathbf{r}_{\mathrm{b}}$ at time $t_{\mathrm{b}}$. The phase shift accumulated along this trajectory is given by

$$
\delta \phi=\frac{M \Omega}{\hbar} \cdot \int_{t_{\mathrm{a}}}^{t_{\mathrm{b}}} \mathrm{d} t[\mathrm{r}(t) \times \mathbf{v}(t)]
$$

But since

$$
\mathrm{d} t \mathbf{v}(t)=\mathrm{d} \mathbf{r}(t)
$$

the phase shift can be rewritten as

$$
\delta \phi=\frac{M \boldsymbol{\Omega}}{\hbar} \cdot \int_{t_{\mathrm{a}}}^{t_{\mathrm{b}}} \mathbf{r}(t) \times \mathrm{d} \mathbf{r}(t)=2 \frac{M \Omega}{\hbar} \cdot \mathcal{A}_{0},
$$

where $\mathcal{A}_{0}$ is the area OAB subtended by the path $\mathbf{r}(t)$ at the origin. 


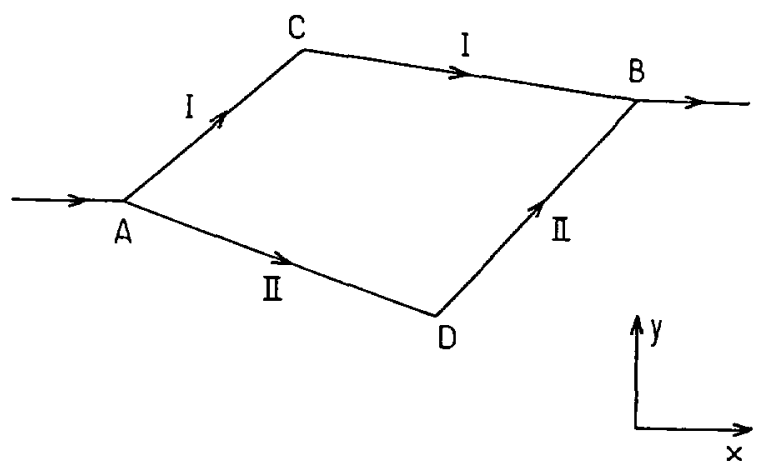

Fig. 12. - Unperturbed paths (in real space) describing the two arms of an atomic interferometer sensitive to rotation.

We now use this result to calculate the phase shift in an interferometer. Since the rotation is just a perturbation on the motion, the results of section 1.3 .5 can be applied, and we obtain

$$
\delta \phi=2 \frac{M \Omega}{\hbar} \cdot \mathcal{A}
$$

where $\mathcal{A}$ is the area ACBDA enclosed by the arms of the interferometer, as shown in figure 12 . Note that like figure 11 , this diagram is in real space rather than spacetime, and the plane shown is perpendicular to the rotation axis.

2.4.3 Comparison with the Sagnac effect. - The Sagnac effect is the phase shift observed in an optical interferometer due to rotation. Here we compare the sensitivity to rotation of optical and matter-wave interferometers.

Consider a rotating circular optical interferometer as shown in figure 13. Two rays emitted from the point $\mathrm{A}$ at time $t=0$ circulate in opposite directions around the interferometer and interfere at the beamsplitter, which is assumed to be at point $B$ at $t=0$. In the absence of rotation the travel times of the two rays to the beamsplitter are equal. However when the interferometer is rotated, the ray travelling in the same sense as $\Omega$ takes a longer time $t^{+}$to reach the beamsplitter according to an observer in a Galilean frame. Likewise a ray travelling in the opposite sense takes a shorter time $t^{-}$The travel times $t^{+}$and $t^{-}$can be determined by the following equations

$$
\begin{aligned}
& c t^{+}=\pi \rho+\rho \Omega t^{+} \\
& c t^{-}=\pi \rho-\rho \Omega t^{-}
\end{aligned}
$$

Hence the time difference is

$$
\begin{aligned}
\Delta t & =t^{+}-t^{-}=\frac{\pi \rho}{c-\rho \Omega}-\frac{\pi \rho}{c+\rho \Omega} \\
& \approx \frac{\pi \rho}{c} \frac{2 \rho \Omega}{c}=\frac{2 \Omega \mathcal{A}}{c^{2}}
\end{aligned}
$$

where $\mathcal{A}=\pi \rho^{2}$ is the area of the interferometer. The Sagnac phase shift is therefore

$$
\delta \phi_{\mathrm{photon}}=\omega_{0} \Delta t=\frac{2 \mathcal{A} \Omega \omega_{0}}{c^{2}}
$$




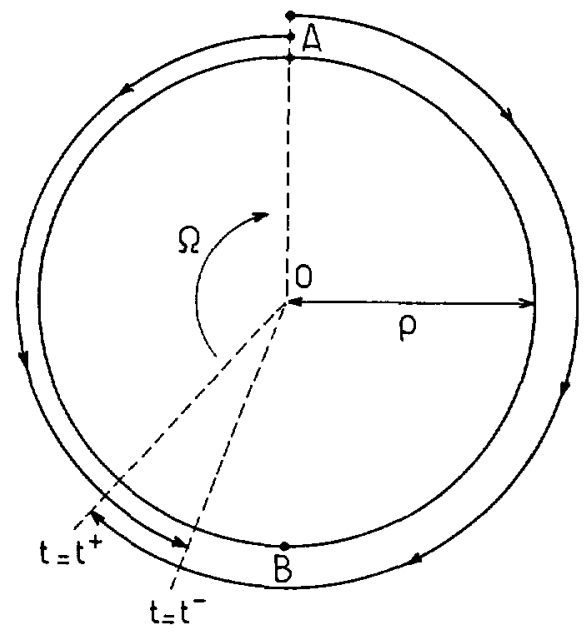

Fig. 13. - Rotating circular optical interferometer. The beamsplitter starts from the point B at time $t=0$, and rotates at angular frequency $\Omega$. The two rays leaving the point $\mathrm{A}$ at $t=0$ circulate in opposite senses and reach the beamsplitter at different times $t^{+}$and $t^{-}$

where $\omega_{0}$ is the (angular) frequency of the light. The relative sensitivity between the optical and matter-wave interferometer for the same enclosed area is

$$
\frac{\delta \phi_{\text {atom }}}{\delta \phi_{\text {photon }}}=\frac{2 \frac{M \mathcal{A} \Omega}{\hbar}}{2 \frac{\mathcal{A} \Omega \omega_{0}}{c^{2}}}=\frac{M c^{2}}{\hbar \omega_{0}} \gg 1 .
$$

From this last result, one might expect to increase the sensitivity by a factor of as much as $10^{10}$ by using matter waves instead of photons. However it must be remembered that the area enclosed by an optical interferometer may be considerably increased over that of a matter wave interferometer by the use of optical fibres. A better signal-to-noise ratio can also be achieved in optical interferometers because of the higher flux of quanta through the apparatus.

2.5 AhARONOV-BoHM EFFECTS. - In this section we will review the scalar and vector Aharonov-Bohm effects $[9,10]$ and describe analogous effects using neutral particles.

2.5.1 The scalar Aharonov-Bohm effect. - Quantum mechanics predicts that a charged particle will be sensitive to an electric potential, even in the absence of any electrical force. The phase shift induced between two wavepackets by subjecting them to different electric potentials is known as the scalar Aharonov-Bohm effect.

The Lagrangian for a charged particle in an electromagnetic field is

$$
L=\frac{1}{2} M \mathbf{v}^{2}+q \mathbf{v} \cdot \mathbf{A}(\mathbf{r})-q U(\mathbf{r}),
$$

where $q$ is the charge on the particle, $U(\mathbf{r})$ is the electric potential, and $\mathbf{A}(\mathbf{r})$ is the vector potential. In the scalar Aharonov-Bohm effect $\mathbf{A}=0$ and $U \neq 0$.

A hypothetical experiment to detect the Aharonov-Bohm effect is indicated in figure 14 . The particle's wavepacket is split into two components I and II by the slits $s_{1}$ and $s_{2}$. Wavepacket I passes through the cylinder $C_{1}$, and wavepacket II through the cylinder $C_{2}$. The two wavepackets subsequently interfere at the point $M$. 


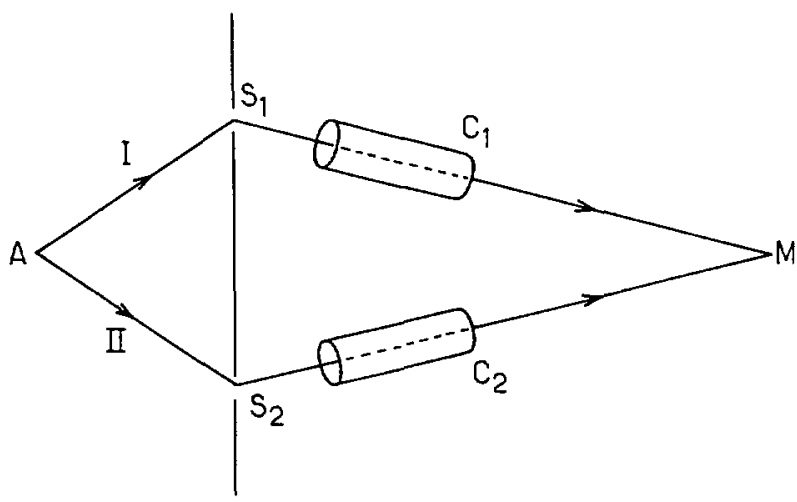

Fig. 14. - Sketch of the experimental setup to test the scalar Aharonov-Bohm effect.

The cylinders are assumed to be longer than the coherence length of the wavepackets, so that, over a certain time interval, the wavepackets will be well localised close to the middle of the cylinders. During this time, the potential $U$ is applied between $C_{1}$ and $C_{2}$ for a duration $T$. Each wavepacket then sees a spatially-uniform potential, and the particle experiences no force. However the potential introduces a phase shift between the two wavepackets of

$$
\delta \phi=\frac{S_{\mathrm{I}}-S_{\mathrm{II}}}{\hbar}=-\frac{q}{\hbar} \oint \mathrm{d} t U=-\frac{q U T}{\hbar},
$$

which results in a displacement of the interference fringes.

2.5.2 The vector Aharonov-Bohm effect. - To test the vector Aharonov-Bohm effect a charged particle is subjected to a magnetic vector potential $\mathbf{A}$. As in the scalar Aharonov-Bohm effect, the particle travels in a region of zero electric and magnetic field, and hence experiences no force.

A possible experimental design is shown in figure 15. The wavepacket of a charged particle is split into two components, which travel around opposite sides of a solenoid before recombining. To ensure that the particle experiences no force, the magnetic field must be entirely confined to the interior of the solenoid. In order to achieve this, the solenoid should in principle be infinitely long or be toroidal in shape. In practice a very long solenoid could alternatively be used.

The phase shift introduced between the two wavepackets by the potential is

$$
\begin{aligned}
\delta \phi & =\frac{S_{\mathrm{I}}-S_{\mathrm{II}}}{\hbar}=\frac{q}{\hbar} \oint \mathrm{d} t \mathbf{v} \cdot \mathbf{A}(\mathbf{r})=\frac{q}{\hbar} \oint \mathrm{d} \mathbf{r} \cdot \mathbf{A}(\mathbf{r}) \\
& =\frac{q}{\hbar} \iint_{\mathcal{A}}[\nabla \times \mathbf{A}(\mathbf{r})] \cdot \mathrm{d} \mathbf{n}=\frac{q}{\hbar} \Phi_{\mathrm{B}}
\end{aligned}
$$

where $\mathcal{A}$ is the surface enclosed by the arms of the interferometer and $\Phi_{\mathrm{B}}$ is the flux of the magnetic field through the solenoid.

The important features of both the scalar and vector Aharonov-Bohm effects are: the absence of force on the particle, the topological nature of the effect, and the fact that the phase shift is global and non-dispersive. This last property means that it is possible to observe fringes even if the length associated with $\delta \phi$ is larger than the coherence length of the wavepacket. 


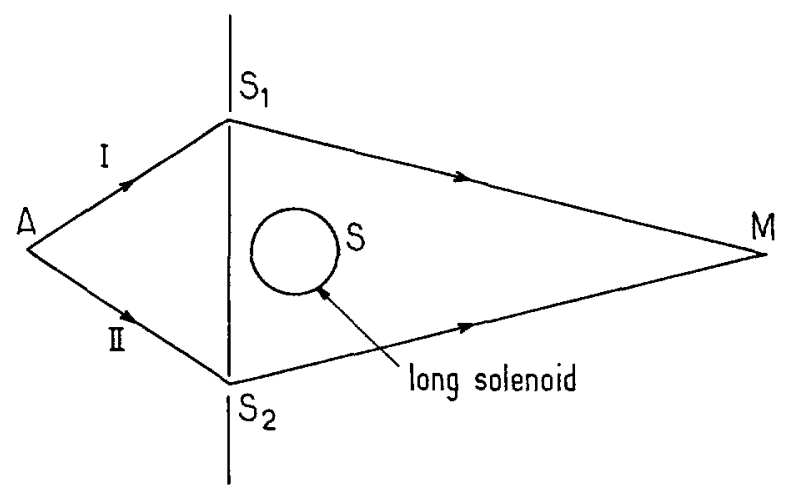

Fig. 15. - Sketch of the experimental setup to test the vector Aharonov-Bohm effect.

2.5.3 Extension to neutral particles. - The scalar Aharonov-Bohm effect, such as it was described above, has not yet been observed. The experiment is very difficult due to the small separation between the interfering beams, the high speeds of the particles, and the technical problems in realizing microcylinders and applying voltages at sufficiently high frequencies (of the order of $\mathrm{GHz}$ ).

The vector Aharonov-Bohm effect has been observed (see [11] and references therein), although the non-dispersive character of the phase-shift has not been tested due to the large coherence lengths of the wavepackets.

In an effort to overcome these problems, new experiments have recently been proposed to generalize both the scalar and vector Aharonov-Bohm effects to neutral particles. Particles with a magnetic moment $\mu$ are coupled to static magnetic or electric fields in which they undergo a phase shift but experience no force.

An equivalent of the scalar Aharonov-Bohm effect was proposed for neutrons by Zeilinger [12]. A beam of neutrons is separated into two components, one of which passes through a solenoid. A current is applied to the solenoid while the wavepacket is well-localised near the centre, giving rise to a potential $-\boldsymbol{\mu} \cdot \mathbf{B}$. The wavefunction undergoes a phase shift, although the particle experiences no force. This proposal has been realized experimentally by Allman et al. [13]. The phase shift observed was in good agreement with the theory, but was not large enough to test the non-dispersive character of the effect. In order to verify this last property, experiments have been performed by Badurek et al. [14] using very slow neutrons. Their experiments however differed from those of Allman et al., in that a single beam of neutrons was used rather than two spatially separated beams. The neutrons were prepared with their polarisation perpendicular to the magnetic field. When the magnetic field was applied the component whose spin was parallel to $\mathbf{B}$ underwent a different phase shift from the component with antiparallel spin. The energy dispersion was high enough and the transit time long enough that even with small magnetic fields it was possible to achieve phase shifts much greater than those corresponding to the coherence length. The non-dispersive character of the phase shift was tested by comparing experiments which used permanent and transitory magnetic fields. Similar experiments using atoms have been performed by Chormaic et al. [15].

The Aharonov-Anandan-Casher effect $[16,17]$ is the equivalent of the vector AharonovBohm effect for neutral particles. The wavepacket of the particle is split into two components which propagate around opposite sides of a long charged rod before recombining. The particle experiences a potential $-\boldsymbol{\mu} \cdot[\mathbf{E}(\mathbf{r}) \times \mathbf{v}] / c^{2}$, although it sees no force. The phase shift acquired 
between the two arms of the interferometer is $\Lambda \mu / \epsilon_{0} \hbar c^{2}$ where $\Lambda$ is the charge per unit length of the rod. The Aharonov-Anandan-Casher effect has been tested experimentally by Cimmino et al. [18] using neutrons, with the charged rod replaced by two pairs of condensing plates. It has also been tested by Sangster et al. [19], using molecules. In their experiment a single beam was passed between two condensing plates. The molecules had been prepared in a superposition of spin states, each of which experienced a different phase shift in the electric field.

In analogy with the Aharonov-Bohm effects, these experiments using neutral particles are characterized by the absence of force, the topological nature of the effect, and the global nondispersive property of the phase shift. However they differ from the Aharonov-Bohm effects in that the particle evolves in regions where the electric and magnetic fields are non-zero.

\section{Conclusion.}

In this paper we have described a path integral approach to solving problems in atomic interferometry. Simple solutions, which offer useful physical insight, have been obtained for situations close to the classical limit when the incident wavefunction can be treated as a plane wave. In particular some exact results are possible when the Lagrangian is a quadratic function of position and velocity, as is the case for a particle in a gravitational field or a rotating reference frame. A simple perturbative treatment has also been presented. We have shown that the phase shift introduced into a wavefunction by a perturbation in the Lagrangian can be calculated by integrating the perturbation along the unperturbed path.

The methods are applicable to a wide variety of current research areas. As examples we have discussed the interferometric measurements of the gravitational acceleration $g$ by Kasevich and $\mathrm{Chu}$, and the atomic equivalents of the Sagnac and Aharonov-Bohm effects.

\section{Acknowledgements.}

We thank Yvan Castin and Jean Dalibard for very fruitful discussions. Pippa Storey is grateful for support from the Fondation Hugot du Collège de France.

\section{Appendix A.}

A.1 Derivation of the Lagrange equations. - Consider a variation $\delta z(t)$ from a classical path $z(t)$ which satisfies the boundary conditions

$$
\delta z\left(t_{\mathrm{a}}\right)=\delta z\left(t_{\mathrm{b}}\right)=0
$$

The path variation changes the values of the Lagrangian and action by the amounts

$$
\begin{aligned}
\delta L & =\frac{\partial L}{\partial z} \delta z(t)+\frac{\partial L}{\partial \dot{z}} \delta \dot{z}(t) \\
\delta S & =\int_{t_{\mathrm{a}}}^{t_{\mathrm{b}}}\left[\frac{\partial L}{\partial z} \delta z(t)+\frac{\partial L}{\partial \dot{z}} \delta \dot{z}(t)\right] \mathrm{d} t .
\end{aligned}
$$

Making the substitution

$$
\delta \dot{z}(t)=\frac{\mathrm{d}}{\mathrm{d} t} \delta z(t)
$$

and integrating by parts gives

$$
\delta S=\left.\frac{\partial L}{\partial \dot{z}} \delta z(t)\right|_{t_{\mathrm{a}}} ^{t_{\mathrm{b}}}+\int_{t_{\mathrm{a}}}^{t_{\mathrm{b}}}\left[\frac{\partial L}{\partial z}-\frac{\mathrm{d}}{\mathrm{d} t} \frac{\partial L}{\partial \dot{z}}\right] \delta z(t) \mathrm{d} t
$$




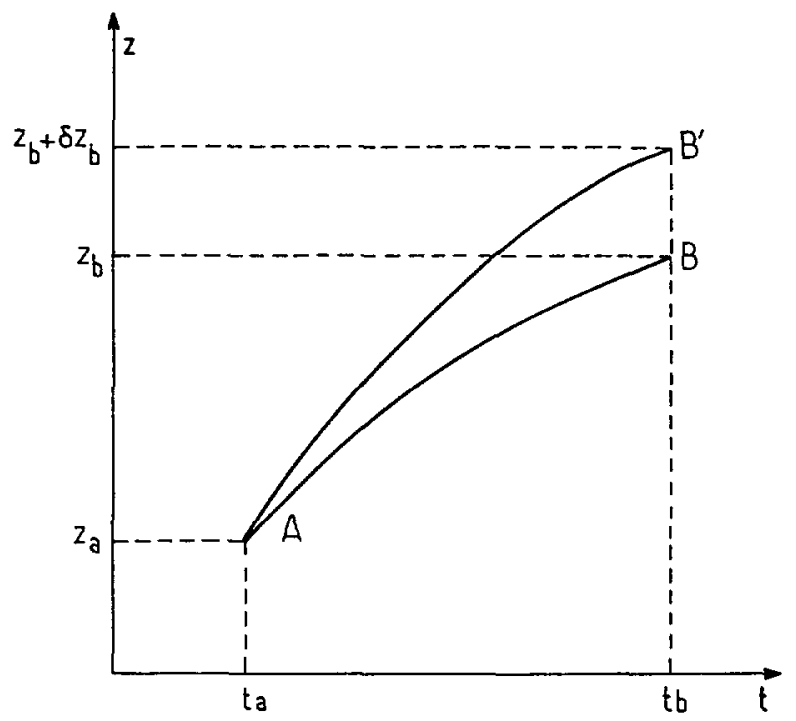

Fig. 16. - Two actual classical paths leave from the same spacetime point A, but arrive at different positions at the final time $t_{\mathrm{b}}$.

Since the action along the actual classical path $z(t)$ is extremal, $\delta S$ must equal zero. The first term in the above equation vanishes due to the boundary conditions (111). The requirement that the second term be zero for arbitrary $\delta z(t)$ gives the Lagrange equations, which describe the dynamics of a classical particle

$$
\frac{\partial L}{\partial z}-\frac{\mathrm{d}}{\mathrm{d} t} \frac{\partial L}{\partial \dot{z}}=0
$$

A.2 The partial Derivatives of THE Classical ACtion.- The classical action is a function of the initial and final times and positions. Here we calculate its partial derivatives with respect to each of these parameters.

A.2.1 Partial derivatives with respect to position.- Consider two actual classical paths (i.e. solutions of the equations of motion) whose final positions differ by $\delta z_{\mathrm{b}}$, as shown in figure 16 . The difference between the actions along these two paths can be calculated using equation (115). Since the paths are classical the second term is identically zero. The first term is calculated using the boundary conditions

$$
\begin{aligned}
& \delta z\left(t_{\mathrm{a}}\right)=0 \\
& \delta z\left(t_{\mathrm{b}}\right)=\delta z_{\mathrm{b}},
\end{aligned}
$$

and gives

$$
\delta S_{\mathrm{cl}}=\frac{\partial L}{\partial \dot{z}} \delta z_{\mathrm{b}}
$$

Using the definition (5) we obtain

$$
\frac{\partial S_{\mathrm{cl}}}{\partial z_{\mathrm{b}}}=p_{\mathrm{b}}
$$

where $p_{\mathrm{b}}$ is the momentum at the final position on the classical path. 


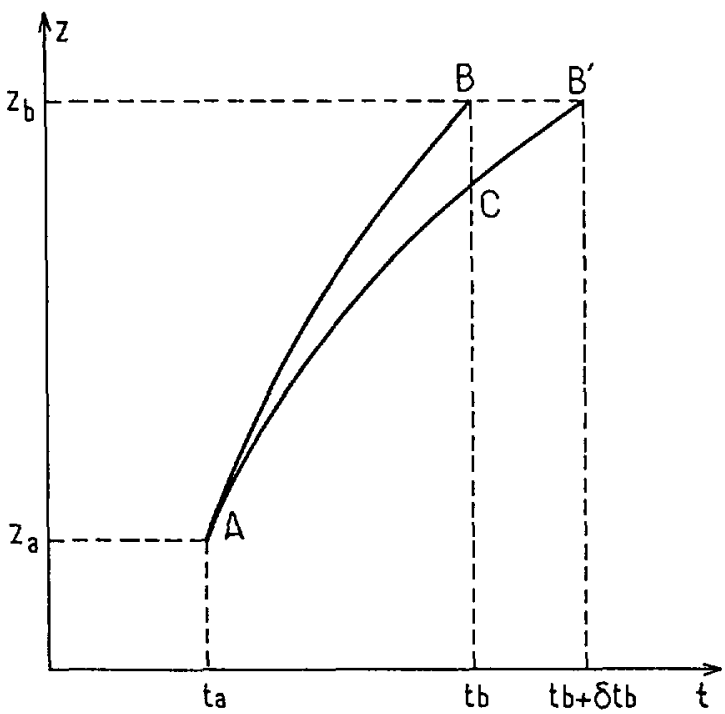

Fig. 17. - Two actual classical paths leave from the same spactime point $A$, but arrive at the final position $z_{\mathrm{b}}$ at different times.

A similar calculation shows that the partial derivative of the classical action with respect to the initial position $z_{\mathrm{a}}$ is

$$
\frac{\partial S_{\mathrm{cl}}}{\partial z_{\mathrm{a}}}=-p_{\mathrm{a}}
$$

where $p_{\mathrm{a}}$ is the momentum at the initial position on the classical path.

A.2.2 Partial derivatives with respect to time. - Consider two actual classical paths whose final times differ by $\delta t_{\mathrm{b}}$ as in figure 17 , and let $\mathrm{C}$ be the point on the path $\mathrm{AB}^{\prime}$ with the same abcissa as $\mathrm{B}$. Then the distance between $\mathrm{B}$ and $\mathrm{C}$ is

$$
\overline{\mathrm{BC}} \approx-\dot{z}_{\mathrm{B}^{\prime}} \delta t_{\mathrm{b}} \approx-\dot{z}_{\mathrm{B}} \delta t_{\mathrm{b}}=-\dot{z}_{\mathrm{b}} \delta t_{\mathrm{b}}
$$

Using equation (120) we find that the difference between the classical actions along the paths $\mathrm{AC}$ and $\mathrm{AB}$ is

$$
S_{\mathrm{AC}}-S_{\mathrm{AB}} \approx p_{\mathrm{b}} \overline{\mathrm{BC}} \approx-p_{\mathrm{b}} \dot{z}_{\mathrm{b}} \delta t_{\mathrm{b}} .
$$

We also know that the difference between the actions along the paths $A B^{\prime}$ and $A C$ is

$$
S_{\mathrm{AB}^{\prime}}-S_{\mathrm{AC}}=S_{\mathrm{CB}^{\prime}} \approx L_{\mathrm{b}} \delta t_{\mathrm{b}}
$$

where $L_{\mathrm{b}}$ is the value of the Lagrangian at the point $\mathrm{B}$ on the path $\mathrm{AB}$.

Adding the above two equations gives the variation in the action due to the change in the final time

$$
\delta S=S_{\mathrm{AB}^{\prime}}-S_{\mathrm{AB}}=-\left(p_{\mathrm{b}} \dot{\mathrm{z}}_{\mathrm{b}}-L_{\mathrm{b}}\right) \delta t_{\mathrm{b}} .
$$

Substituting the definition (8) of the Hamiltonian into the above equation gives

$$
\frac{\partial S_{\mathrm{cl}}}{\partial t_{\mathrm{b}}}=-H_{\mathrm{b}}
$$


A similar calculation shows that the partial derivative of the classical action with respect to the initial time is

$$
\frac{\partial S_{\mathrm{cl}}}{\partial t_{\mathrm{a}}}=H_{\mathrm{a}} .
$$

\section{References}

[1] Special issue of Applied Physics B: Optics and Interferometry with Atoms, Volume B54, J. Mlynek, V. Balykin and P. Meystre Eds. (May 1992).

[2] C. Bordé, Phys. Lett. A140 (1989) 10; Laser Spectroscopy X, M. Ducloy, E. Giacobino and G. Camy Eds. (World Scientific 1992) p. 239.

[3] Landau L.D. and Lifshitz E.M., Mechanics (Pergamon Press, Oxford, 1960).

[4] Feynman R.P., Rev. Mod. Phys. 20 (1948) 367.

[5] Feynman R.P. and Hibbs A.R., Quantum Mechanics and Path Integrals (McGraw Hill, New York, 1965).

[6] Poston T. and Stewart I., Catastrophe Theory and its Applications (Pitman, 1978).

[7] Berry M.V., Proc. Phys. Soc. 89 (1966) 479;

Berry M.V. and Mount K.E., Rep. Prog. Phys. 35 (1972) 315.

[8] Kasevich M. and Chu S., Appl. Phys. B54 (1992) 321.

[9] Aharonov Y. and Bohm D., Phys. Rev. 115 (1959) 485.

[10] Furry W.H. and Ramsey N.F., Phys. Rev. 118 (1960) 623.

[11] Tonomura A., Osakabe N., Matsuda T., Kawasaki T., Endo J., Yans S. and Yamada H., Phys. Rev. Lett. 56 (1986) 792.

[12] Zeilinger A., J. Phys. Colloq. France 45 (1984) C3-213.

[13] Allman B.E., Cimmino A., Klein A.G., Opat G.I., Kaiser H. and Werner S.A., Phys. Rev. Lett. 71 (1993) 307; Phys. Rev. A48 (1993) 1799.

[14] Badurek G., Weinfurter H., Gähler R., Kollmar A., Wehinger S. and Zeilinger A., Phys. Rev. Lett. 71 (1993) 307.

[15] Nic Chormaic S., Miniatura Ch., Gorceix O., Viaris de Lesegno B., Robert J., Feron S., Lorent V., Reinhardt J., Baudon J, and Rubin K., Phys. Rev. Lett. 72 (1994) 1.

[16] Aharonov Y. and Casher A., Phys. Rev. Lett. 53 (1984) 319.

[17] Anandan J., Phys. Rev. Lett. 48 (1982) 1660.

[18] Cimmino A., Opat G.I., Klein A.G., Kaiser H., Werner S.A., Arif M. and Clothier R., Phys. Rev. Lett. 63 (1989) 380.

[19] Sangster K., Hinds E.A., Barnett S. and Riis E., Phys. Rev. Lett. 71 (1993) 3641. 